\title{
The essentials of the mode-coupling theory for glassy dynamics
}

\author{
W.Götze \\ Physik-Department, Technische Universität München, \\ D-85747 Garching, Germany
}

Received April 16, 1998

The essential results of the mode-coupling theory for the evolution of structural relaxation in simple liquids such as the Debye-Waller-factor anomaly, the critical decay, von Schweidler's law, the $\boldsymbol{\alpha}$ - and $\boldsymbol{\beta}$-relaxation scaling, the appearance of two divergent time scales, and Kohlrausch's law for the $\alpha$-process are explained, and their relevance to the understanding of experiments in glass-forming systems is described.

Key words: mode-coupling theory, structural relaxation, glassy dynamics, bifurcation dynamics

PACS: 64.70.Pf, 61.20.LC

In memoriam: I first met Dmitrii Nikolaevich Zubarev in 1969, when I came to Moscow to work with his team at the Steklov-Institute. Since then we met regularly. I always enjoyed discussing with him many open problems in statistical physics and I learned a lot from his unbiased and well contemplated views on various issues. I like to remember his gentleness, his warm hospitality, and the conversations with him on non-scientific matters. I dedicate this article to the memory of Dmitrii Nikolaevich with gratitude.

\section{Introduction}

Usually a liquid transforms in a solid if it is condensed by cooling or compression. There are several possibilities for the solid state. It may be, for example, a crystalline solid characterized by an arrangement of molecules with a long-ranged order. It may also be an amorphous solid, called glass, where all spatial correlations decay rapidly for large separations. As a precursor of the glass formation there appears an anomalous dynamics, called the glassy dynamics. It exhibits features which are utterly different from those known for other states of condensed matter. Some aspects of this anomalous dynamics will be discussed in this article. 
Three outstanding features of the glassy dynamics have been known for more than a century [1]. First, the characteristic time scale $\tau$ of the slowest relaxation process can be many orders of magnitude larger than the time scale $\tau_{\text {mic }}$, which characterizes the conventional microscopic motion. The latter is about 1 picosecond. The spectra for the normal state of conventional condensed matter are located in the THz-band; they are observed, for example, by Raman-scattering or neutron-scattering experiments. In supercooled liquids $\tau$ can be 100 seconds or larger, i.e. $\tau$ can exceed $\tau_{\text {mic }}$ by 14 or more orders of magnitude. Second, $\tau$ depends sensitively on control parameters, such as temperature $T$ and density $n$. A decrease of $T$ by one degree may lead to an increase of $\tau$ by the factor 10 .

The third and most puzzling feature is the stretching of the glassy dynamics over huge windows. Relaxation of some disturbance may require an increase of time $t$ by several orders of magnitude for the decay from, say, $90 \%$ to $10 \%$ of the initial value. Similarly, one has to increase frequency $\omega$ by several decades to scan the upper $90 \%$ of a typical bump of a susceptibility spectrum. This stretching makes a comprehensive measurement of the glassy dynamics very difficult.

The mentioned first two properties imply that for a cooling experiment there is a reasonably well defined temperature $T_{\mathrm{g}}$, where relaxation time $\tau$ equals time $\tau_{\text {exp }}$, characterizing the experiment: $\tau\left(T=T_{\mathrm{g}}\right)=\tau_{\text {exp }}$. For $T<T_{\mathrm{g}}$ the system is quenched in a history-dependent-non-equilibrium state. This state is glass in the technical sense of this word. Since $T_{\mathrm{g}}$ is usually measured by scanning calorimetry, it is called the calorimetric glass-transition temperature. $T_{\mathrm{g}}$ depends on the scanning speed and, therefore, it does not play a role in the equilibrium theory of matter. The following discussion is restricted to the amorphous regime $T>T_{\mathrm{g}}$, where all structure functions vary smoothly with control parameters and where classical physics can be used for the description of the matter.

The best technique for the study of the glassy dynamics, which was available to the pioneers, is a dielectric-loss spectroscopy. Until very recently it could be used to measure spectra for frequencies around a $\mathrm{GHz}$ and smaller. With the changes of $T$ the spectra shift through the whole accessible dynamical window, and they are as puzzling for $\omega$ near $1 \mathrm{GHz}$ as they are for $\omega$ near $0.01 \mathrm{~Hz}$ [2]. Despite many efforts it was not possible to suggest a coherent physical picture for the anomalous dynamics, leave aside a theory [1].

Since the glassy dynamics is absent in the THz-band but present in cooled liquids for all frequencies below $1 \mathrm{GHz}$, it must evolve upon cooling within the GHzband. Obviously, one expects that studying the evolution of the glassy dynamics within the GHz-band can reveal to us some essential features of this phenomenon. Beginning with neutron scattering work $[3,4]$ it became possible during the past ten years to explore anomalous dynamics in the $\mathrm{GHz}$-band and, indeed, a whole series of new phenomena has been discovered. Furthermore, a microscopic theory for the evolution of the glassy dynamics, called the mode-coupling theory (MCT), was proposed, whose results correlate reasonably with some of the experimental facts. These new approaches towards an ancient problem led to a lively discussion of the glass-transition problem, which is reflected in the proceedings of various 
conferences [5-7]. In this paper some of the non-trivial and surprising results of the MCT will be described and their relation to some of the modern experiments on the evolution of the glassy dynamics will be sketched.

\section{The basic version of the mode-coupling theory}

The distribution in space of $\mathrm{N}$ particles with positions $\vec{r}_{\alpha}, \alpha=1, \ldots, N$, can be described in terms of the density fluctuations of wave vector $\vec{q}, \rho_{\vec{q}}=\sum_{\alpha} \exp \left(\mathrm{i} \vec{q} \vec{r}_{\alpha}\right)$, and products thereof. The simplest function characterizing the equilibrium structure is the structure factor $S_{q}=\left\langle\left|\rho_{\vec{q}}\right|^{2}\right\rangle / N$; here $\langle\ldots\rangle$ denotes canonical averaging and $q=|\vec{q}|$ abbreviates the wave-vector modulus. In classical systems, as opposed to the systems where quantum mechanics has to be used, all structure functions are determined by the ratio of potential and thermal energy; they are independent of inertia parameters such as the particle masses. The simplest functions dealing with the structure dynamics are density correlators $\phi_{q}(t)=\left\langle\rho_{\vec{q}}(t)^{*} \rho_{\vec{q}}\right\rangle /\left\langle\left|\rho_{\vec{q}}\right|^{2}\right\rangle$; they can be measured, for example, by neutron-spin-echo or by photon-correlation spectroscopy. Such auto-correlation functions of variables $A, \phi_{A}(t)=\left\langle A(t)^{*} A\right\rangle$, are positive-definite functions. Their Fourier-cosine transforms $\phi_{A}^{\prime \prime}(\omega)$, the fluctuation spectra determine the Fourier-Laplace transforms $\phi_{A}(\omega)=\phi_{A}^{\prime}(\omega)+\mathrm{i} \phi_{A}^{\prime \prime}(\omega)$ by the Kramers-Kronig relation [8]. The density spectrum $\phi_{q}^{\prime \prime}(\omega)$ can be measured by a neutron-scattering or a light-scattering experiment. The dynamical susceptibility of variable $A$ is given by $\chi_{A}(\omega)=\left(\omega \phi_{A}(\omega) / k_{\mathrm{B}} T+\chi_{A}^{T}\right)$, where $\chi_{A}^{T}$ denotes the thermodynamic susceptibility $\left\langle|A|^{2}\right\rangle / k_{\mathrm{B}} T$. In particular, there is a trivial relation between the fluctuation spectrum and the susceptibility spectrum: $\omega \phi_{q}^{\prime \prime}(\omega) / k_{\mathrm{B}} T=\chi_{q}^{\prime \prime}(\omega)$. The density spectrum can be characterized by an averaged frequency $\Omega_{q}$, defined by the sum rule: $\Omega_{q}^{2}=\int \omega^{2} \phi_{q}^{\prime \prime}(\omega) \mathrm{d} \omega / \int \phi_{q}^{\prime \prime}(\omega) \mathrm{d} \omega$. It is expressible as $\Omega_{q}=v q / \sqrt{S_{q}}$, where $v$ denotes thermal velocity.

Within the Zwanzig-Mori formalism one can derive the equation of motion

$$
\ddot{\phi}_{q}(t)+\Omega_{q}^{2} \phi_{q}(t)+\int_{0}^{t} M_{q}\left(t-t^{\prime}\right) \dot{\phi}_{q}\left(t^{\prime}\right) \mathrm{d} t^{\prime}=0 .
$$

This is an oscillator equation where the influence of the non-trivial interaction effects are expressed in terms of a retarded friction quantified by the memory kernel $M_{q}(t)$. This kernel can be written as a canonical average of some fluctuating force $F_{\vec{q}}(t): M_{q}(t)=\left\langle F_{\vec{q}}^{*}(t) F_{\vec{q}}\right\rangle[8]$. Within the MCT the kernel is split into a regular part $M_{q}^{\mathrm{reg}}(t)$ and the main contribution $\Omega_{q}^{2} m_{q}(t)$. The former is assumed to describe the conventional liquid dynamics and the latter is anticipated to be due to the slowly fluctuating forces caused by the slowly moving structure:

$$
M_{q}(t)=M_{q}^{\mathrm{reg}}(t)+\Omega_{q}^{2} m_{q}(t) .
$$

The preceding equations can be rewritten in an equivalent form in the frequency domain as a formula for the susceptibility:

$$
\chi_{q}(\omega) / \chi_{q}^{T}=-\Omega_{q}^{2} /\left\{\omega^{2}-\Omega_{q}^{2}+\omega\left[M_{q}^{\mathrm{reg}}(\omega)+\Omega_{q}^{2} m_{q}(\omega)\right]\right\} .
$$


This shows that $\chi_{q}(\omega)$ can be viewed as a phonon propagator, where $\Omega_{q}$ plays the role of a bare phonon frequency. The interaction of the bare phonon with other dynamical variables of the system is expressed in terms of the polarization operator $M_{q}(\omega)=M_{q}^{\mathrm{reg}}(\omega)+\Omega_{q}^{2} m_{q}(\omega)$. Given the regular kernel $M_{q}^{\mathrm{reg}}(t)$, equations $(1)$ are an exact rewriting of the problem. The question of determining $\phi_{q}(t)$ is shifted to that of calculating $m_{q}(t)$.

Interaction forces occur between pairs of particles, and, therefore, a contribution to $F_{\vec{q}}(t=0)$ is a combination of density fluctuation pairs $\rho_{\vec{p}} \rho_{\vec{k}}$ with $\vec{p}+\vec{k}=\vec{q}$. Treating the force correlator with a factorization approximation, introduced by Kawasaki in some other context [9], one gets

$$
m_{q}(t)=\sum_{\vec{k}+\vec{p}=\vec{q}} V(\vec{q} ; \vec{k} \vec{p}) \phi_{k}(t) \phi_{p}(t) .
$$

An important finding is, that vertices $V(\vec{q} ; \overrightarrow{k p})$ are given by the structure factors:

$$
V(\vec{q} ; \overrightarrow{k p})=n S_{q} S_{k} S_{p}\left\{\vec{p}\left[\vec{k} C_{k}+\vec{p} C_{p}\right]\right\}^{2} /\left(2 q^{4}\right) .
$$

Here $C_{q}$ denotes a direct correlation function related to the structure factor by the Ornstein-Zernike relation $S_{q}=1 /\left[1-n C_{k}\right]$. The potential does not occur explicitly but only indirectly via $S_{q}$. Within the amorphous state, $S_{q}$ and hence the vertices $V(\vec{q} ; \overrightarrow{k p})$ are smoothly varying functions of the wave vectors and all the control parameters. They can be evaluated with a reasonable accuracy using some standard theory for $S_{q}$. The sums over $\vec{k}$ and $\vec{p}$ in equation (2a) can be converted into a double integral over $k$ and $p$. For calculational convenience the wave vector can be discretized to a set of $M$ values, so that one finds

$$
\begin{gathered}
m_{q}(t)=\mathcal{F}_{q}\left(\phi_{k}(t)\right), \\
\mathcal{F}_{q}\left(f_{k}\right)=\sum_{k, p=1}^{M} V_{q, k p} f_{k} f_{p},
\end{gathered}
$$

and a trivial relation between $V_{q, k p} \geqslant 0$ and the vertices in equation ( $\left.2 \mathrm{~b}\right)$. Equations (3) formulate a coupling of the fluctuating forces to the density modes. Therefore, $\mathcal{F}_{q}$ is called a mode-coupling functional. Equations (3) are equivalent to the formula relating the spectrum of the polarization-kernel contribution $m_{q}(\omega)$ to the densityfluctuation spectra:

$$
\pi m_{q}^{\prime \prime}(\omega)=\sum_{k, p=1}^{M} \int \mathrm{d} \omega_{1} \int \mathrm{d} \omega_{2} V_{q, k p} \delta\left(\omega-\omega_{1}-\omega_{2}\right) \phi_{k}^{\prime \prime}\left(\omega_{1}\right) \phi_{p}^{\prime \prime}\left(\omega_{2}\right) .
$$

Thus, the mode-coupling contribution to the kernel spectrum $M_{q}^{\prime \prime}(\omega)$ accounts for the decay of the phonon of frequency $\omega$ and wave vector $q$ into all pairs of phonons characterized by wave vectors $k$ and $p$ and frequencies $\omega_{1}$ and $\omega_{2}$, respectively. Equations (1-3) are the basis of the MCT. One can generalize the theory to a 
treatment of mixtures $[10,11]$ or of non-spherical molecules $[12,13]$, but these extensions will not be considered in the following. Given the structure factor $S_{q}$ and some model for $M_{q}^{\text {reg }}(t)$, the MCT equations are closed and allow the evaluation of correlators $\phi_{q}(t)$ for all the control parameters.

The cited equations were first derived and solved numerically - for their quantum-mechanical generalization - in order to calculate the dynamical structure factor $S_{q}(\omega)=\phi_{q}^{\prime \prime}(\omega) S_{q}$ of liquid helium II at zero temperature [14]. From this work one infers, that the MCT equations deal with an approximation for the backflow phenomenon, which is known to be the essence of the roton spectrum in helium. The derivation of equations $(1,2)$ for simple classical systems was done by Sjögren within a generalized kinetic theory [15]. From his work and some other related works one knows that the MCT deals with a cage effect which is an outstanding feature distinguishing a simple classical liquid from a dense gas. If the nearest neighbours of a tagged particle were fixed, the particle would be trapped in a cage. It could only rattle within the cage but it could not move over distances of the order of the particle diameter. In order to move over longer distances the cage has to be opened and this requires the cooperative rearrangement of many other particles. The dynamics in the liquid deals with the interplay of the motion in cages and the motion of the cage boundaries. The relevance of the MCT to the explanation of the glassy dynamics was noticed in 1984 by Leutheusser [16] and by Bengtzelius et al. [17]. A review of the derivation of the cited formulae, a derivation of similar equations for the tagged particle motion and shear, and a discussion of their implications for hydrodynamic phenomena can be found in [18].

For some of the following discussions it is not necessary to appreciate the microscopic background of the MCT. One can use equations $(1,3)$ as a description of a mathematical model for dynamics. It aims at the evaluation of the $M$ variables $\phi_{q}(t)$ for $t>0$ which obey the initial conditions $\phi_{q}(t=0)=1, \dot{\phi}_{q}(t=0)=0$. The model is specified by frequencies $\Omega_{q}>0$, positive-definite functions $M_{q}^{\text {reg }}(t)$, and by non-negative coupling constants $V_{q, k p}$. The question is: how do the solutions vary if the coupling constants are changed? There is also interest in the dependence of the solutions on $\Omega_{q}$ and $M_{q}^{\text {reg }}(t)$; but in this paper such questions will not be considered. One can show the following $[19,20]$. The equations have a unique solution $\phi_{q}(t), q=1, \ldots, M$. The solutions $\phi_{q}(t)$ are positive-definite functions, i.e. they have the standard properties of correlators. The solutions depend smoothly on $\Omega_{q}, M_{q}^{\text {reg }}(t)$ and $V_{q, k p}$ for all finite time intervals. Hence, equations $(1,3)$ are a proper definition of a mathematical theory.

Obviously, MCT does not anticipate any properties of the glassy dynamics, singularities, power laws and the like. In the following no additional hypothesis will be introduced, but merely mathematical implications of equations (1-3) will be reported. The MCT deals with non-linear equations. The essential difference from standard theories for a non-linear dynamics is the appearance of retardation effects, given by the convolution integral in equation (1a). The microscopic features are embedded into the mathematical model by specifying the coupling constants $V_{q, k p}$ in terms of the physical control parameters $n$ and $T$ via equations (2). 
The simplest model from a conceptual point of view is obtained by using $M_{q}^{\text {reg }}(t)=0$. It treats density fluctuations as free oscillators which are coupled by the mode-coupling kernels $m_{q}(t)$. It handles a conventional liquid so as if only the cage effect would be relevant. Comprehensive studies of this oscillator model are not yet available, but some numerical solutions can be found in [21,22]. The simplest model from a mathematical point of view is obtained if the regular term is replaced by a Newtonian friction, $\int_{0}^{t} M_{q}^{\text {reg }}\left(t-t^{\prime}\right) \dot{\phi}_{q}\left(t^{\prime}\right) \mathrm{d} t^{\prime}=\nu \dot{\phi}_{q}(t), \nu>0$, and if in addition the inertia term is neglected. Then, equation (1a) is simplified to

$$
\tau_{q} \dot{\phi}_{q}(t)+\phi_{q}(t)+\int_{0}^{t} m_{q}\left(t-t^{\prime}\right) \dot{\phi}_{q}\left(t^{\prime}\right) \mathrm{d} t^{\prime}=0
$$

where the time constants are given by $\tau_{q}=\nu / \Omega_{q}$. This is a model for a colloid, i.e. for a system of macroscopic particles dispersed in some solvent. Notice that for a given interparticle interaction the structure factor $S_{q}$, and hence the modecoupling functional $\mathcal{F}_{q}$, of the colloid model is the same as for the oscillator model. In the latter model the microscopic motion is determined by Newton's equations of motion. For the colloid model the microscopic dynamics is the Brownian motion. For the colloid model one can show that the solutions are completely monotone, i.e. $\left(-\partial_{t}\right)^{\ell} \phi_{q}(t)>0$ for $\ell=0,1, \ldots$ [20]. In [20] one can find a representative set of solutions for a simple example for equation (4) and [23] contains a discussion of the solutions for a hard-sphere-colloid model.

\section{Glass-transition singularities}

The asymptotic values $f_{q}=\phi_{q}(t \rightarrow \infty)$ obey $0 \leqslant f_{q} \leqslant 1$. They describe the long time limit $\delta \rho_{\vec{q}}^{\infty}$ of the density fluctuation $\delta \rho_{\vec{q}}(t)$, which was created at time zero as $\delta \rho_{\vec{q}}^{0}: \delta \rho_{\vec{q}}^{\infty}=f_{q} \delta \rho_{\vec{q}}^{0}$. These numbers $f_{q}$ can be evaluated straightforwardly from the mode-coupling functional as follows. The set of $M$ coupled implicit equations $\tilde{f}_{q} /\left(1-\tilde{f}_{q}\right)=\mathcal{F}_{q}\left(\tilde{f}_{k}\right)$ has a maximum solution $f_{q}^{\max }$ in the sense that $f_{q}^{\max } \geqslant$ $\tilde{f}_{q}, q=1, \ldots, M$, for all the possible solutions $\tilde{f}_{q}$. The sequence $f_{q}^{\ell}$, obtained by the iteration $f_{q}^{\ell+1} /\left(1-f_{q}^{\ell+1}\right)=\mathcal{F}_{q}\left(f_{k}^{\ell}\right), f_{q}^{0}=1, \ell=0,1,2, \ldots$ converges monotonically towards $f_{q}^{\max }: f_{q}^{\ell} \geqslant f_{q}^{\ell+1} \rightarrow f_{q}^{\max }[20]$. The maximum solution is the long time limit: $f_{q}=f_{q}^{\max }[18]$.

For sufficiently small couplings one finds the trivial solution $f_{q}=0$. In this case the density perturbations $\delta \rho_{\vec{q}}(t)$ disappear if one waits long enough. Such behaviour is expected for a liquid. However, for sufficiently large coupling constants $V_{q, k, p}$ one finds a non-trivial solution where, generically, for $q=1, \ldots, M: f_{q}>0$. In this case perturbations do not disappear; the perturbed system does not return to equilibrium. The density spectrum exhibits an elastic spike on a continuous background: $\phi_{q}^{\prime \prime}(\omega)=\pi f_{q} \delta(\omega)+\cdots$. The existence of such a spike is one sign of the solid state, where $f_{q}$ is called the Debye-Waller factor. The long-ranged order in a crystalline solid is reflected by the fact that its Debye-Waller factor is non-zero only on a discrete set of reciprocal-lattice vectors. For the present problem, if the original situation of a continuous wave vector is considered, $f_{q}$ varies 
smoothly with $q$. Thus, strong-coupling solutions describe amorphous solids, i.e. glass states. The Debye-Waller factor $f_{q}$ is also referred to as a glass form factor or a non-ergodicity parameter. Edwards and Anderson pointed out in the discussion of a spin-glass problem that the existence of a non-zero long-time limit of some correlator, $\phi_{A}(t \rightarrow \infty)=f_{A}>0$, is the sign of an ideal glass state [24]. Hence, the specified MCT-strong-coupling solutions are ideal glass states in the sense of Edwards and Anderson, where $A=\rho_{\vec{q}}$. The form factors $f_{q}$ are, therefore, also called the Edwards-Anderson parameters.

If the coupling constants are increased smoothly from small to large values, there must occur singularities for the long-time limits $f_{q}$. Such a phenomenon is called bifurcation [25]. Within the MCT the positions of these bifurcations in the parameter space are referred to as glass-transition singularities. A mode-coupling functional without linear terms, as exemplified by equation (3b), implies that $f_{q}$ changes discontinuously at the singularity from zero to the positive value $f_{q}^{c}>0$. $f_{q}^{c}$ is called a critical form factor. The appearance of the described ideal liquidto-glass transition is a strong-coupling feature which cannot be anticipated from perturbation expansions with respect to the mode-coupling effects. It results from the feed-back mechanism between force fluctuations, described by the kernel $m_{q}(t)$, and density fluctuations, described by the correlators $\phi_{q}(t)$, which is anticipated by the self-consistent treatment of these two functions. It was described first in [26] how this self-consistency problem leads to a transition from the solutions $\phi_{q}(t)$, which have a trivial long-time limit $f_{q}=0$, to those which have the positive form factor $f_{q}$. This precursor theory was applied, for example, to a discussion of the transition from the Lorentz-liquid to the Lorentz-glass $[27,28]$.

One can show $[18,20]$ that the MCT bifurcations are of the cuspoid type denoted by Arnol'd as $A_{\ell}$ [25]. If we imagine the coupling constants to be smooth functions of a control parameter, say of the density $n$, the generic singularity at some critical point $n_{\mathrm{c}}$ is a fold bifurcation $A_{2}$ [25]. Let us characterize the neighbourhood of the singularity by the distance parameter $\epsilon=\left(n-n_{\mathrm{c}}\right) / n_{\mathrm{c}}$. The critical value $n_{\mathrm{c}}$ separates the liquid states for $\epsilon<0$ from the glass states for $\epsilon \geqslant 0$. Having determined $n_{\mathrm{c}}$ and $f_{q}^{\mathrm{c}}$, it is straightforward to calculate from the mode-coupling functional the so-called guage constant $C>0$, the number $\lambda$ between $1 / 2$ and 1 , and the so-called critical amplitude $h_{q}>0$, so that for $\epsilon \geqslant 0$ [18]:

$$
f_{q}=f_{q}^{\mathrm{c}}+h_{q} \sqrt{\sigma /(1-\lambda)}+O(\epsilon), \quad \sigma=C \epsilon .
$$

The function $\sigma$ is called a separation parameter. If the transition is driven by temperature, one gets the same result with $\epsilon=\left(T_{\mathrm{c}}-T\right) / T_{\mathrm{c}}$, except that the gauge constant $C$ is to be changed. Let us also note that formula (5) can be generalized within the microscopic MCT to evaluate the long-time limit $f_{A}$ for the correlator $\phi_{A}(t)$ of any variable $A$, which couples to density fluctuations. In equation (5) merely $f_{q}^{c}, h_{q}$ have to be replaced by the corresponding $A$-specific positive numbers $f_{A}^{c}, h_{A} ; \sigma$ and $\lambda$ remain unchanged.

The archetype of a model for classical matter is the hard-sphere system (HSS), dealing with hard spheres of diameter $d$. Temperature $T$ does not enter the struc- 
ture functions for the HSS. The density $n$ is the only physical control parameter which is usually quoted as the packing fraction $\varphi=n d^{3} \pi / 6$. The structure factor $S_{q}$ can be evaluated with reasonable accuracy analytically within the Percus-Yevick theory. The MCT result for the critical packing fraction for a liquid-to-glass transition is $\varphi_{\mathrm{c}} \sim 0.52$, and the critical form factor was predicted to oscillate between 0.4 and 0.9 if the wave vector varies between $q d \sim 5$ and $q d \sim 10$ [17]. An ideal glass transition was identified and studied extensively by van Megen, Pusey and Underwood for a hard sphere colloid using photon-correlation spectroscopy, as can be inferred from [29] and the citations given in this paper. An elucidatory summary of this work is given in [30]. The experimental value for the critical packing fraction is 0.58 and the measured critical Debye-Waller factor $f_{q}^{c}$ agrees within the experimental uncertainty with the predicted one. Kob and Andersen [31-33] performed detailed molecular-dynamics studies for a binary mixture and thereby they identified the critical coupling constant $\Gamma_{\mathrm{c}}$ and determined three independent critical glass-form factors as functions of $q$. The MCT calculations for their model [34] showed again that the theory somewhat overestimates the trend to glass formation, and that the form factors agreed with the data within $10 \%$. It appears non-trivial that the MCT is able to determine a condensed-matter quantity like $f_{q}^{\mathrm{c}}$ so well.

The first system, for which a form factor anomaly in agreement with equation (5) was detected convincingly, is the van der Waals liquid orthoterphenyl (OTP). Using incoherent-neutron-scattering experiments for a series of representative wave vectors, Petry et al. identified the square-root anomaly with $T_{\mathrm{c}} \sim 290 \mathrm{~K}$ [35]. This temperature is located between the calorimetric glass transition temperature $T_{\mathrm{g}}=$ $243 \mathrm{~K}$ and the melting temperature $T_{\mathrm{m}}=329 \mathrm{~K}$. Later experiments by coherentneutron scattering, which are summarized in [36], corroborated the finding. The impulsive-stimulated-light-scattering spectroscopy was developed by Nelson and Yang to a technique for accurate measuring the Debye-Waller factor for small $q$. Formula (5) was shown to be compatible with the data for several systems. For example, the critical temperature $T_{\mathrm{c}} \sim 380 \mathrm{~K}$ was identified for the mixed salt $0.4 \mathrm{Ca}\left(\mathrm{NO}_{3}\right)_{2} 0.6 \mathrm{KNO}_{3}\left(\mathrm{CKN} ; T_{\mathrm{g}}=333 \mathrm{~K}, T_{\mathrm{m}}=438 \mathrm{~K}\right)$ [37]. It came as a surprise that the predicted square-root anomaly was found for standard systems like OTP or CKN within an easily excessible and often studied temperature range.

\section{The two time fractals}

For parameters off the glass-transition singularities the correlators approach their long-time asymptote exponentially [20], and this implies a regular low-frequency fluctuation spectrum: $\phi_{q}^{\prime \prime}(\omega)=O\left(\omega^{0}\right)$. Such frequency-independent fluctuation spectrum, also called white noise, is equivalent to the linear susceptibility spectrum $\chi_{q}^{\prime \prime}(\omega)=O(\omega)$. However, at the glass-transition singularities the correlators decay towards their long-time plateau $f_{q}^{c}$ algebraically:

$$
\phi_{q}\left(\hat{t}_{0}\right)=f_{q}^{\mathrm{c}}+h_{q} \hat{t}^{-a}+O_{q}\left(\hat{t}^{-2 a}\right), \quad \varphi=\varphi_{\mathrm{c}} .
$$


The time scale $t_{0}$ is determined by the transient motion. The quantities $f_{q}^{\mathrm{c}}, h_{q}$ are the same as those introduced in equation (5). The anomalous exponent $a$ is to be calculated from the previously mentioned $\lambda$ by the equation $\Gamma(1-a)^{2} / \Gamma(1-2 a)=$ $\lambda, 0<a \leqslant 1 / 2$, where $\Gamma$ denotes a gamma function. Therefore, $\lambda$ is called an exponent parameter. The result (6) is referred to as the critical decay.

Since the solutions $\phi_{q}(t)$ depend smoothly on $\varphi$ for every finite time interval, one concludes: there is a characteristic time scale $t_{\sigma}$, so that equation (6) holds for $t_{0} \ll$ $t \ll t_{\sigma}$, also for $\varphi \neq \varphi_{\mathrm{c}}$, except for corrections of order $\epsilon$. In this sense the critical decay is $\epsilon$-insensitive. In particular, the law holds for $\varphi \geqslant \varphi_{\mathrm{c}}$, as well as for $\varphi \leqslant \varphi_{\mathrm{c}}$. Obviously, $t_{\sigma} \rightarrow \infty$ for $\sigma \rightarrow 0$. The sensitivity of the solution $\phi_{q}(t)$ with respect to control-parameter variations is hidden in the time scale $t_{\sigma}$, deliminating the window for the applicability of the leading-order-asymptotic formula $\phi-f^{c}=h \hat{t}^{-a}$. The critical law implies a low-frequency enhancement of the fluctuation spectrum above the white noise level: $\phi_{q}^{\prime \prime}\left(t_{\sigma}^{-1} \ll \omega \ll t_{0}^{-1}\right) \propto 1 / \omega^{1-a}$, or equivalently, the sublinear susceptibility spectrum $\chi_{q}^{\prime \prime}\left(t_{\sigma}^{-1} \ll \omega \ll t_{0}^{-1}\right) \propto \omega^{a}$.

The existence of the described critical decay process was not noticed in the classical literature on the glassy dynamics. The prediction of the critical spectral enhancement for $T$ near $T_{\mathrm{c}}$ and for frequencies just below the microscopic excitation band, $\omega t_{0} \ll 1$, provided a rather specific motivation to measure spectra in glassy systems within the GHz-window. The critical spectrum was first identified for the mentioned mixed salt CKN. Neutron-scattering spectra, testing density fluctuations for microscopic distances [38], polarized-light-scattering spectra [39], testing fluctuations for macroscopic distances, and depolarized-light-scattering data [39] showed the spectral enhancement compatible with equation (6) for $a=0.30 \pm 0.05$. Recently a break through in the dielectric-loss spectroscopy was achieved, allowing one to use this classical probing of the dynamics in the full $\mathrm{GHz}$-window. This can be inferred from [40] and the papers quoted there. For CKN the cited power law $\omega^{0.3}$ was also confirmed for the dielectric function [41]. Most impressive are the dielectric-loss spectra for $0.4 \mathrm{Ca}\left(\mathrm{NO}_{3}\right)_{2} \quad 0.6 \mathrm{RbNO} \mathrm{N}_{3}$. They exhibit the critical law with the critical exponent $a=0.20$ for $T=361 \mathrm{~K}$ for a large window extending from $0.3 \mathrm{GHz}$ up to $300 \mathrm{GHz}$ [41]. For the HSS the exponent parameter was calculated as $\lambda \approx 0.76$ [42]. This implies $a=0.30$, a result which fits reasonably the van Megen-Pusey experiments for hard-sphere-colloid glass [43,44].

For small negative $\epsilon$ and $t>t_{\sigma}$, the correlators decay from $f_{q}^{c}$ to zero. A characteristic time scale $\tau$ for this process can be defined, for example, by $\phi_{q}(\tau)=$ $f_{q}^{\mathrm{c}} / 2$. Obviously, $\tau$ has to diverge for $\varphi \rightarrow \varphi_{\mathrm{c}}-$. The mentioned slow and controlparameter-sensitive decay process in the liquid is viewed as the analogue of the glassy-relaxation process mentioned in section 1. Following the terminology of the classical literature [1], it is referred to as an $\alpha$-process. For the initial part of this $\alpha$-process one finds another algebraic law:

$$
\phi_{q}\left(\tilde{t}_{\sigma}^{\prime}\right)=f_{q}^{\mathrm{c}}-h_{q} \tilde{t}^{b}+O_{q}\left(\tilde{t}^{2 b}\right), \quad \varphi \rightarrow \varphi_{\mathrm{c}}-.
$$

The $\alpha$-process leads to a bump of the susceptibility spectrum $\chi_{q}^{\prime \prime}(\omega)$, called an $\alpha$-peak. The position of the peak maximum $\omega_{\max }$ is of the order $1 / \tau$. Equation 
(7a) is equivalent to the power-law decay of the high-frequency wing of the $\alpha$ peak: $\chi_{q}^{\prime \prime}\left(\tilde{\omega} / t_{\sigma}^{\prime} \gg 1\right) \propto 1 / \tilde{\omega}^{b}$. The anomalous exponent $b$ is also determined by the exponent parameter: $\lambda=\Gamma(1+b)^{2} / \Gamma(1+2 b), 0<b \leqslant 1$. The time scale $t_{\sigma}^{\prime}$, which is to be discussed more explicitly below, agrees with $\tau$ up to an $\epsilon$-independent factor. Let us note that within the MCT the equations $(6,7 \mathrm{a})$ can also be generalized to asymptotic laws for the correlators $\phi_{A}(t)$ of other variables $A$ coupling to the density. One merely has to replace the critical Debye-Waller factor $f_{q}^{c}$ and the critical amplitude $h_{q}$ by the corresponding quantities $f_{A}^{\mathrm{c}}$ and $h_{A}$, respectively. The numbers $t_{0}, a, b, t_{\sigma}, t_{\sigma}^{\prime}$ remain the same [18].

It was already noticed in 1907 by von Schweidler that many data for dielectric relaxation can be fitted by equation (7a), and, therefore, this result was named after him. A verification of von Schweidler's law for $T>T_{\mathrm{c}}$ for the dynamical window as large as three decades was published for the mentioned simulation data for a mixture [31]. In this case the optimal fit to the correlation functions gave an exponent $b=0.49$. Molecular-simulation results for the incoherent-intermediatescattering function for supercooled water have been shown to follow the von Schweidler-law extension $\phi_{q}(t)=f_{q}^{c}-h_{q}(t / \tau)^{b}+k_{q}(t / \tau)^{2 b}, b \approx 0.5$, over a large $q$-range and a considerable temperature interval [45]. The cited photon-correlation data for a supercompressed hard-sphere colloid [43] exhibit von Schweidler's law with the calculated exponent $b \approx 0.54$, as it is shown in [44].

A discussion of the range of validity of the specified power laws can be based on the evaluation of the leading corrections, i.e. of the prefactors of the $\hat{t}^{-2 a}$ and $\tilde{t}^{2 b}$ terms in equations (6,7a). Such results can be inferred from [23] together with detailed demonstrations for the HSS and a list of citations of the earlier theoretical work. Obviously, the range of validity of von Schweidler's law is restricted to not too small times, as well as to not too large times, as it will be shown more precisely in section 7 below. Here it will be emphasized only that equation (7a) is to be understood as an exact implication of the MCT equations in the sense of a double limit, where the sequence of limits must not be interchanged:

$$
\lim _{\tilde{t} \rightarrow 0} \lim _{\epsilon \rightarrow 0-}\left[\phi_{q}\left(\tilde{t} t_{\sigma}^{\prime}\right)-f_{q}^{c}\right] / \tilde{t}^{b}=-h_{q} .
$$

A fold bifurcation for a conventional dynamics, i.e. for a set of coupled ordinary differential equations, also leads to equation (6) albeit with the universal normal exponent $a=1$. This result does not change if one introduces retarded friction specified by the integrable kernel $M_{q}(t)$. In this case there is an effective finite length $\tau_{r}$ for the relevant retardation times $\left(t-t^{\prime}\right)$ in the integral of equation (1a). As soon as the decay time $\tau$ exceeds $\tau_{r}$, one can replace $\int_{0}^{t} M_{q}\left(t-t^{\prime}\right) \dot{\phi}_{q}\left(t^{\prime}\right) \mathrm{d} t^{\prime}$ by $\dot{\phi}(t) \nu_{q}$, where $\nu_{q}=\int_{0}^{\infty} M_{q}\left(t-t^{\prime}\right) \mathrm{d} t^{\prime}$. Since $\tau \rightarrow \infty$ for $\varphi \rightarrow \varphi_{\mathrm{c}}$, the retardation effects become irrelevant near the transition. However, in the MCT the retardation time $\tau_{\mathrm{r}}$ is not a fixed number: The kernel $M_{q}(t)$ is given by equation (2a). Substituting there equation (6) leads to $M_{q}\left(t_{0} \hat{t}\right)=M_{q}^{\mathrm{c}}+H_{q}^{\mathrm{c}} \hat{t}^{-a}+O\left(\hat{t}^{-2 a}\right)$ for $\varphi=\varphi_{\mathrm{c}}$ with $M_{q}^{\mathrm{c}}>0$ and $H_{q}^{\mathrm{c}}>0$. Hence, in the MCT: $\tau_{\mathrm{r}} \rightarrow \infty$ for $\varphi \rightarrow \varphi_{\mathrm{c}}$. It is the interplay of non-linearities with divergent retardation times $\tau_{\mathrm{r}}$ which leads to the anomalous critical exponent $a$. 
The value of $\lambda$, and hence of $a$ and $b$, are the same for all the functions $\phi_{A}$, $M_{q}(t), \phi_{q}(t)$ referring to the given mode-coupling functional $\mathcal{F}_{q}$. However, different systems are specified by different $\mathcal{F}_{q}$. This will imply, in general, different values for $\lambda$ and, hence, for the anomalous exponents. In this sense the critical exponent $a$ and von Schweidler's exponent $b$ are not universal.

A critical decay law, specified by the anomalous exponent $a$, occurs also at second order phase-transition points. It was Kawasaki's achievement to evaluate critical laws within a mode-coupling theory [9]. But his well established theory differs in many respects from the theory discussed here. For phase-transitions there is a critical cluster in configuration space and this leads to small- $q$ divergencies of the equilibrium-structure factors. The divergency of the thermodynamic susceptibilities like $S_{q}$, leads to divergencies in the mode-coupling-functional vertices. These small $q$ divergencies depend on the universal exponents of the susceptibilities. Furthermore, the special form of the hydrodynamic singularities of the correlators can be relevant to the critical dynamics. Therefore, the dynamical exponent $a$ in phase-transition theories is universal, but systems from the same universality class for the equilibrium structure can lead to different universality classes for dynamics. Furthermore, for phase-transitions the critical dynamics depends singularly on the wave vector. Contrary to what is anticipated for phase transitions, the input information $S_{q}$ for the present MCT does not exhibit singularities. The mode-coupling functional does not have relevant small $q$ divergencies. The dominant contributions to the mode-coupling integrals come from wave vector ranges near the inverse interparticle distance. Therefore, $\lambda$ depends on microscopic details of the structure and the exponents are not universal. The wave vector does not enter critically in the results, but merely modulates smoothly the parameters $f_{q}^{\mathrm{c}}$ and $h_{q}$ in equations $(6,7)$. In the MCT there is no space fractal underlying the found two time fractals. The exponents neither depend on the transient motion as quantified by $\Omega_{q}$ and $M_{q}^{\mathrm{reg}}(t)$ nor on the type of hydrodynamics considered for the system. There is no analogue for von Schweidler's law in the theory of phase transitions.

The rather transparent integro-differential equations $(1,3)$ formulate a novel paradigm for the appearance of time fractals. In particular, the MCT has delivered a derivation of von Schweidler's law within a microscopic theory of liquids. Equation (7a) plays a crucial role in Lévy's theory of stable probability distributions. This theory aims at a generalization of the central limit theorem to those cases where the variance of the probability densities does not exist [46]. Interpreting $\phi_{q}(t)$ as a characteristic function of probability distributions, the MCT has established a connection between the microscopic theory of glassy systems and a branch of the probability theory.

\section{The first scaling law}

One can solve the MCT equations of motion by asymptotic expansion, using $\delta \phi_{q}(t)=\phi_{q}(t)-f_{q}^{c}$ as a small quantity. In the leading order one finds the factorization of $\delta \phi_{q}(t)$ in the critical amplitude $h_{q}$ and in the function $G(t)$, which 
describes the time-and-control-parameter dependence. The result can be generalized as mentioned above for correlators of any variable $A$ coupling to the density fluctuations:

$$
\phi_{A}(t)=f_{A}^{\mathrm{c}}+h_{A} G(t)+O\left(\delta \phi_{q}(t)^{2}\right) .
$$

This formula is an analogue of the centre manifold theorem of the conventional bifurcation theory. The domain of times $t$ or of frequencies $\omega$, where the leading order expression $\phi_{q}(t)=f_{q}+h_{q} G(t)-$ or $\chi_{q}^{\prime \prime}(\omega)=h_{q} \chi^{\prime \prime}(\omega), \chi^{\prime \prime}(\omega)=\omega G^{\prime \prime}(\omega)-$ is valid, is also referred to as the MCT- $\beta$-relaxation window; and then $G(t)$ and $\chi^{\prime \prime}(\omega)$ are called $\beta$-correlator and $\beta$-susceptibility spectrum, respectively. Within this window the problem of solving the $M$ coupled integro-differential equations $(1,3)$ is reduced to solving one equation for $G(t)$; and this reads:

$$
\sigma+\lambda G(t)^{2}=(\mathrm{d} / \mathrm{d} t) \int_{0}^{t} G\left(t-t^{\prime}\right) G\left(t^{\prime}\right) \mathrm{d} t^{\prime} .
$$

It is complemented by the initial condition $G(t \rightarrow 0) \sim\left(t_{0} / t\right)^{a}$. Equation (9) can be solved analytically on a $2 \%$ accuracy level by various asymptotic expansions, and an efficient algorithm for the numerical solution is also available $[47,48]$.

Function $G$, which depends for the given exponent parameter $\lambda$ on time $t$ and on the separation parameter $\sigma$, obeys the scaling law:

$$
\begin{aligned}
& G(t)=c_{\sigma} g_{-}\left(t / t_{\sigma}\right) \quad \text { for } \quad \sigma<0, \\
& =\left(t_{0} / t\right)^{a} \text { for } \quad \sigma=0, \\
& =c_{\sigma} g_{+}\left(t / t_{\sigma}\right) \quad \text { for } \quad \sigma>0 \text {. }
\end{aligned}
$$

Here the correlation scale is the square-root law characteristic of the fold bifurcation: $c_{\sigma}=\sqrt{|\sigma|}$. The qualitative significance of the time scale $t_{\sigma}$ was mentioned in the preceding section; it also obeys a power law

$$
t_{\sigma}=t_{0} /|\sigma|^{1 / 2 a}
$$

The master functions $g_{ \pm}(\hat{t})$ are given by $\lambda$. They are independent of the control parameters and are to be evaluated from equation (9) for $\sigma= \pm 1$, respectively. For short rescaled times $\hat{t}=t / t_{\sigma}$ the $\beta$-correlator does not discriminate between glass and liquid: $\lim _{\hat{t} \rightarrow o} \hat{t}^{a} g_{ \pm}(\hat{t})=1$. The glass correlator exhibits arrest for times exceeding $t_{\sigma}: \lim _{\hat{t} \rightarrow \infty} g_{+}(\hat{t})=1 / \sqrt{1-\lambda}$, and this reproduces equation (5). For long rescaled times $\hat{t}$ the liquid correlator exhibits von Schweidler's law: $\lim _{\hat{t} \rightarrow \infty} g_{-}(\hat{t}) / \hat{t}^{b}=-B$. Here $B$ is some positive constant given by $\lambda$. This result can be combined with equation (8) to reproduce equation (7a) with the power law time scale

$$
t_{\sigma}^{\prime}=t_{0} B^{-1 / b} /|\sigma|^{\gamma}, \quad \gamma=(1 / 2 a)+(1 / 2 b) .
$$

The cited leading-order-asymptotic results establish universality features of the MCT-bifurcation dynamics. The evolution of the dynamics near the ideal liquidto-glass transition is given by universal functions $g_{ \pm}$and by universal scales. There 
enter only time constant $t_{0}$, specifying the microscopic dynamics, and guage constant $C$ relating the relevant control parameter $\sigma$ to the external control parameter $\epsilon$, equation (5). In addition there appear two amplitudes $f_{A}, h_{A}$, which characterize function $A$ used as a probe for dynamics. This holds for all the systems exhibiting the same exponent parameter $\lambda$. Different universality classes are specified by different $\lambda$, but all the results depend smoothly on $\lambda$. The result (10) is called the first scaling law of the MCT. It deals with the dynamics near the transition within a mesoscopic window where time is large relative to scale $t_{0}$ for the transient, but small compared with scale $t_{\sigma}^{\prime}$ for the $\alpha$-process. The range of validity of the leading-order formula can be determined by calculating the leading corrections. These are not universal in the sense that the corrections are determined by two additional amplitudes and two new constants, all to be calculated from the mode-coupling functional $\mathcal{F}_{q}$. For the given $\sigma$ and for the given dynamical window it can happen, that the results of the leading-plus-next-to-the leading asymptotic formulae disagree with the leading-order results even qualitatively. Details can be found in [23] together with quantitative examples for the HSS and citations of the earlier literature.

Formula (8) implies that density fluctuations $\delta \rho_{\vec{r}}(t)$ vary according to the law $\delta \rho_{\vec{r}}(t)-\delta \rho_{\vec{r}}^{\mathrm{c}}=H(\vec{r}) G(t)$. Here $\delta \rho_{\vec{r}}^{\mathrm{c}}$ and $H(\vec{r})$ are the Fourier back transformations of $f_{q}^{\mathrm{c}} \delta \rho_{\vec{q}}^{(0)}$ and $h_{q} \delta \rho_{\vec{q}}^{(0)}$, respectively, with $\delta \rho_{\vec{q}}^{(0)}$ denoting the Fourier transform of the initial perturbance $\delta \rho_{\vec{r}}^{0}$. Hence, within the $\beta$-relaxation window, variations of the positions $\vec{r}$ and time $t$ are uncorrelated. In this sense the excitations can be viewed as localized. The first tests of this factorization property, supporting the MCT prediction, were done for the molecular dynamics data for a binary mixture of soft spheres [49] and for a model of CKN [50]. Kob and Anderson [32] demonstrated the factorization property for the relevant window $t_{0}<t<t_{\sigma}^{\prime}$, which was nearly as large as three decades. They also demonstrated that the factorization does not hold for their system for the major part of the $\alpha$-process, i.e. for $t \geqslant t_{\sigma}^{\prime}$, nor for the transient dynamics, i.e. for $t \leqslant t_{0}$. The cited tests are restricted to $T>T_{\mathrm{c}}$. Unfortunately, so far simulation data could not be obtained for temperatures at and below the critical $T_{\mathrm{c}}$.

The complete scenario for the evolution of the glassy dynamics was studied for the first time by $\mathrm{Li}$ et al. [51] by depolarized-light scattering for CKN. By combining the results from a Raman spectrometer with those from a tandemFabry-Pérot spectrometer, these experiments were also the first to map out the dynamics of a condensed matter system within the full GHz-band. The authors concluded that their data for the temperatures between $T_{\mathrm{g}}$ and $T_{\mathrm{g}}+135 \mathrm{~K}$ and for the frequencies between $0.2 \mathrm{GHz}$ and $200 \mathrm{GHz}$ could be interpreted consistently by the cited leading-order asymptotic MCT formulae. For example, for $T>380 \mathrm{~K}$ the susceptibility spectra $\chi^{\prime \prime}(\omega)$ exhibited a minimum at some position $\omega_{\min }$, where the spectral intensity $\chi_{\text {min }}$ was up to two orders of magnitude larger than it was expected for a white-noise background. This spectrum could be described by the $T$-independent master susceptibility $\chi_{-}^{\prime \prime}(\hat{\omega})$. This $\beta$-relaxation susceptibility for a liquid deals with the crossover from the von Schweidler susceptibility proportional 
to $\left(\omega t_{\sigma}^{\prime}\right)^{-b}$ to the critical susceptibility proportional to $\left(\omega t_{0}\right)^{a}$. The quantitative description of the spectra holds, provided $\lambda=0.81$ was chosen, implying the MCT exponents of CKN: $a=0.273, b=0.459,1 / 2 a=1.8, \gamma=2.9$. The plot of $\chi_{\min }^{2}$ and $\omega_{\min }^{2 a}$ versus $T$ gave two straight lines with a common intersection of the abscissa at the critical temperature $T_{\mathrm{c}}$. The third straight line, also intersecting the abscissa near $T_{\mathrm{c}}=380 \mathrm{~K}$, was obtained for an $\omega_{\max }^{1 / \gamma}$ versus $T$ plot, where $\omega_{\max } \propto 1 / t_{\sigma}^{\prime}$ denotes an $\alpha$-peak position of the susceptibility spectrum. The found value $T_{\mathrm{c}}$ is consistent with the one obtained by Yang and Nelson [37] for the Debye-Wallerfactor anomaly. It was shown in addition $[52,53]$ that the identified $\beta$-correlator $G(t)$ could account for the decay curves obtained for CKN by neutron spin echo measurements [54], a result supporting the factorization theorem (8). The dielectric loss spectra measured for $T>T_{\mathrm{c}}$ also follow the scaling law predictions with the cited value for $\lambda$ [41]. Let us note that for the quoted data analysis those and only those numbers $T_{\mathrm{c}}, \lambda, t_{0}$ etc. were used as fit parameters, which the MCT introduced as necessary for the specification of the leading-order-asymptotic formulae.

After identification of the general formulae for the asymptotic dynamics one can answer the question: what are the simplest models reproducing the essence of the theory? Obviously, the simplest model deals with a single correlator only, say $\phi(t)$. A simple model for the regular kernel in equation (1b) leads to Newtonian friction, so that the Zwanzig-Mori equation of motion reads:

$$
\ddot{\phi}(t)+\nu \dot{\phi}(t)+\Omega^{2} \phi(t)+\Omega^{2} \int_{0}^{t} m\left(t-t^{\prime}\right) \dot{\phi}\left(t^{\prime}\right) \mathrm{d} t^{\prime}=0 .
$$

A simple example of the mode-coupling functional is a polynomial of degree two:

$$
m(t)=v_{1} \phi(t)+v_{2} \phi(t)^{2} .
$$

The bifurcation equation $f /(1-f)=v_{1} f+v_{2} f^{2}$ defines a line for the transition in the $v_{1}-v_{2}$ plane for two coupling constants. Every point on this line yields some exponent parameter $\lambda$, so that the line can be parametrized by $\lambda$. One gets: $v_{1}^{\mathrm{c}}=(2 \lambda-1) / \lambda^{2}, v_{2}^{\mathrm{c}}=1 / \lambda^{2}, 0.5 \leqslant \lambda<1[18,55]$. Thus, this model is the simplest example which can reproduce all the values of the exponents. Specifying one exponent, the transition point is fixed for this model. There is no freedom left to vary, e.g., the amplitudes. One gets the critical form factor $f^{\mathrm{c}}=1-\lambda$ and the critical amplitude $h=\lambda$; in particular, $f^{\text {c }}$ is smaller than $1 / 2$. A model, which allows one to choose an arbitrary value for the critical form factor can be obtained by introducing a second correlator, say $\phi_{\mathrm{s}}(t)$. It obeys the same equation of motion (12a) as the first one with $\nu, \Omega$ and $m(t)$ replaced by $\nu_{\mathrm{s}}, \Omega_{\mathrm{s}}$ and $m_{\mathrm{s}}(t)$, respectively. A simple kernel specified by a single additional coupling constant $v_{\mathrm{s}}$ reads [56]:

$$
m_{\mathrm{s}}(t)=v_{\mathrm{s}} \phi(t) \phi_{\mathrm{s}}(t) .
$$

The peculiarity of a specified two component model is, that the first correlator influences the second, but the second has no effect on the first. Therefore, the exponent parameter is not changed. One gets for the second the critical form factor 
and the critical amplitude, respectively: $f_{\mathrm{s}}^{\mathrm{c}}=1-\left(1 / v_{\mathrm{s}} f^{\mathrm{c}}\right), h_{\mathrm{s}}=\lambda /\left[v_{\mathrm{s}} f^{\mathrm{c} 2}\right]$, which can be regulated by varying $v_{\mathrm{s}}$. Such simple ad hoc models, referred to as schematic models, clearly demonstrate that the subtleties of the MCT dynamics are not due to the complexities of the liquid structure, of hydrodynamic phenomena and the like, but rather due to the interplay of non-linearities with retardation effects.

\section{The second scaling law and Kohlrausch's law}

Let us come back to the $\alpha$-process, i.e. to the decay of the liquid correlators $\phi_{q}(t)$ below the plateau $f_{q}^{c}$ for $t>t_{\sigma}$. For this process one can prove the asymptotic law: $\lim _{\sigma \rightarrow 0-} \phi_{q}\left(\tilde{t}_{\sigma}^{\prime}\right)=\tilde{\phi}_{q}(\tilde{t})$. Here $\tilde{\phi}_{q}$ is a control-parameter-independent master function obeying the initial condition $\tilde{\phi}_{q}(\tilde{t})=f_{q}^{c}-h_{q} \tilde{t}^{b}+O\left(\tilde{t}^{2 b}\right)$. It can be obtained as a solution of a set of $M$ coupled implicit functional equations. These equations are specified by the mode-coupling functional $\mathcal{F}_{q}^{\mathrm{c}}$ at the glass-transition singularity, i.e. by equation (3b) with coupling constants $V_{q, k p}$ evaluated at the critical point [18]:

$$
\begin{gathered}
\int_{0}^{t}\left[N_{q}\left(t^{\prime}\right)-\tilde{\phi}_{q}\left(t^{\prime}\right)\right] \mathrm{d} t^{\prime}=\int_{0}^{t} N_{q}\left(t-t^{\prime}\right) \tilde{\phi}_{q}\left(t^{\prime}\right) \mathrm{d} t^{\prime}, \\
N_{q}(t)=\mathcal{F}_{q}^{\mathrm{c}}\left(\tilde{\phi}_{k}(t)\right) .
\end{gathered}
$$

Thus, for sufficiently small separations $|\sigma|$, the liquid correlators obey the second scaling law:

$$
\phi_{q}(t)=\tilde{\phi}_{q}\left(t / t_{\sigma}^{\prime}\right) .
$$

Thereby the slowness and control-parameter sensitivity of the slowest MCT process is explained by the divergence of the scale $t_{\sigma}^{\prime}$, equation (11b). The singular variation of $t_{\sigma}^{\prime}$ causes via equation (14) a singular dependence of the $\alpha$-process on $T, n$ and possibly on other external variables. The scaling law (14) has been often described in the classical literature [1] where it is referred to as the time-temperaturesuperposition principle. Formula (14) is equivalent to the expression for the $\alpha$-peaksusceptibility spectra: $\chi_{q}^{\prime \prime}(\omega)=\tilde{\chi}_{q}^{\prime \prime}\left(\omega \cdot t_{\sigma}^{\prime}\right)$. The master spectra $\tilde{\chi}_{q}^{\prime \prime}(\tilde{\omega})$ are related, as usual, to the Fourier-cosine transforms $\tilde{\phi}_{q}^{\prime \prime}(\tilde{\omega})$ of $\tilde{\phi}_{q}(\tilde{t}): \tilde{\chi}_{q}^{\prime \prime}(\tilde{\omega})=\tilde{\omega} \tilde{\phi}_{q}^{\prime \prime}(\tilde{\omega})$.

The interval for the separations $|\sigma|$, where the second scaling law is valid, is larger than the one where the first scaling law holds. This comes about because the relative sizes of the corrections to the cited leading-asymptotic formulae are proportional to $|\sigma|$ for law (14), while they vary proportionally to $\sqrt{|\sigma|}$ for law (10). Upon increasing $|\sigma|$, the corrections to the superposition principle start to become important for short rescaled times $\tilde{t}=t / t_{\sigma}^{\prime}$ or large rescaled frequencies $\tilde{\omega}=\omega t_{\sigma}^{\prime}$, respectively. These corrections are due to the $\beta$-relaxation process and can be calculated in the leading order in terms of the asymptotic limits of this process. Thus, one understands that the range of validity of equation (14) is rather large and one also understands quantitatively the deviations from this asymptotic result. These findings are described and illustrated in detail for the HSS in [23]. Upon heating the system at $T>T_{\mathrm{c}}$, the $\alpha$-peaks of the susceptibility spectra 
$\chi_{q}^{\prime \prime}(\omega)$ shift to higher frequencies. According to the scaling law, the position of the peak maximum varies like $\omega_{\max } \propto 1 / t_{\sigma}^{\prime}$. At very high temperatures the peaks merge with the band of microscopic excitations. The $\alpha$-peak is superimposed on the critical spectrum and, therefore, the peak height increases somewhat with increasing the temperature. But the merging of the $\alpha$-peak is not accompanied by a serious variation of the peak shape, in particular, there is no serious decrease of the $\alpha$-peak stretching due to the increase of $T$.

The described scenario is found in the molecular-dynamics-studies of [33] for a binary mixture and of [57] for supercooled water. The studies for the hard sphere colloids [29] have shown that the data are in agreement with equation (14), where the predicted HSS-master-functions $\tilde{\phi}_{q}[58]$ were used. In addition, it was demonstrated [29] that the small- $\tilde{t}$ corrections to the leading-order result (14) agreed with the terms expected from the $\beta$-process. The $\alpha$-peaks for the susceptibility spectra, determined by depolarized-light-scattering for OTP, verified the superposition principle for temperatures up to $80 \mathrm{~K}$ above the melting temperature [59].

Elementary theories, developed in 1867 by Maxwell within his approach towards visco-elastic effects and by Debye in 1929 for the dipole motion in fluids, represent the slowest relaxation process by the exponential $\phi_{\mathrm{D}}(t)=f \exp [-(t / \tau)]$. This is equivalent to the Lorentzian susceptibility spectrum $\chi_{\mathrm{D}}^{\prime \prime}=f \omega \tau /\left[1+(\omega \tau)^{2}\right]$. Function $\phi_{\mathrm{D}} / f$ decays from 0.9 to 0.1 if $t / \tau$ increases by the factor 23 , i.e. if the time varies over 1.4 decades. Correspondingly, the half-width at half-height of $\chi_{\mathrm{D}}^{\prime \prime}$ is 1.1 decade. However, this Maxwell-Debye description does not cope with the facts in glassy liquids. It was already pointed out in section 1 that in glassy systems decay curves and susceptibility spectra are stretched over larger dynamical windows of two or more decades [1]. The MCT-master-functions $\tilde{\phi}_{q}$ are not universal; they depend via equations $(2 \mathrm{~b}, 13 \mathrm{~b})$ on the details of the structure on microscopic length scales. And the dynamics described by the master function $\tilde{\phi}_{q}(\tilde{t})$ is stretched. The von Schweidler fractal is the generic reason for stretching the MCT$\alpha$-process. An efficient algorithm to solve equations (13) for shape functions can be found in [60]. It is also shown there that rather simple schematic models produce master spectra in perfect agreement with the presented experimental findings. A detailed discussion of the theoretical $\alpha$-relaxation master functions for the HSS is presented in [58].

Relaxation stretching was discovered already in 1854 by Kohlrausch. He reported that his dielectric-polarization-decay data can be described well, though not perfectly, by the stretched exponential: $\exp \left(-\tilde{t}^{\beta}\right), \beta<1$. In our century Kohlrausch's law has been used regularly as a reasonable fit of the major part of the $\alpha$-process. But it has also been often reported that the data exhibit small systematic deviations from this law, as can be inferred from the review [61]. Indeed, it is impossible that Kohlrausch's law describes all the correlators of a given system. For example, the exact equation of motion (1) is not compatible with a simultaneous representation of the elastic modulus and of the density correlator by the Kohlrausch function [21]. In general, the MCT solutions do not reproduce the Kohlrausch law, even though, for example, the master functions for the schematic 
model, defined by equations (12a,b), are very close to it [60].

It is a remarkable discovery by Fuchs [62] that the solutions of the microscopic MCT equations (13) yield the Kohlrausch function as an asymptotic result in the limit of large wave-vectors. With the time parameter $\tilde{\tau}_{q}=\left(f_{q} / h_{q}\right)^{1 / b}$ he could show:

$$
\lim _{q \rightarrow \infty} \tilde{\phi}_{q}\left(t^{*} \tilde{\tau}_{q}\right) / f_{q}^{\mathrm{c}}=\exp \left(-t^{* b}\right) .
$$

The correlators outside the transient regime can be interpreted as characteristic functions of probability distributions. For large $q$ the MCT equations obtain correlators as sums of many small terms, each of which exhibits the von Schweidlershort-time asymptote, equation (7a). Therefore, Lévy's limit theorem [46] suggests the characteristic function of a stable distribution as a sum, and this is Kohlrausch's law with $\beta=b$. For wave vectors of the order of the reciprocal interparticle distance or smaller, the MCT equations imply strong correlations between the functions $\phi_{q}(\tilde{t})$ referring to different $q$. In this case various contributions to the correlator are not independent, thus the cited limit theorem cannot be applied and the correlators deviate from the Kohlrausch function. If one approximates the solutions as $\tilde{\phi}_{q}(\tilde{t}) \propto \exp \left[-\left(t / \tau_{q}\right)^{\beta_{q}}\right]$, the fit parameter $\beta_{q}$ varies with $q$ and differs from $b$. The result (15) leads to the prediction $\lim _{q \rightarrow \infty} \beta_{q}=b$ supported by the molecular-dynamics-simulation data which were obtained by Sciortino et al. [57] for supercooled water.

Hence, the MCT leads to a microscopic derivation of the oldest law formulated for the dynamics of glassy systems. It provided an explanation why the $\alpha$-relaxation of those variables $\mathrm{A}$, which are composed of many small independent density fluctuations of large wave vectors, obeys this law. It also explained why the spectra generically do not follow Kohlrausch's law precisely.

\section{The two critical time scales}

Three time scales $t_{0}, t_{\sigma}$ and $t_{\sigma}^{\prime}$ are used above to quantify the MCT-bifurcation dynamics. The time $t_{0}$ specifies the scale for transient motion; $1 / t_{0}$ characterizes the position of the frequencies for the normal condensed matter excitations. $t_{0}$ depends on $\Omega_{q}$ and $M_{q}^{\mathrm{reg}}(t)$ in equations (1). It also depends on the influence of the cage effect on the normal liquid dynamics as described by the kernel $m_{q}(t)$. For small separation parameters $|\sigma|$, to which the following considerations will be restricted, the precise definition of $t_{0}$ is given by equation (6) for the critical decay. One can show that the dynamics for $t \gg t_{0}$, including its variation due to changes of the control parameters like $T$ and $n$, depends on the transient only via $t_{0}: \phi_{q}(t)=F_{q}\left(t / t_{0}\right)$. Here $F_{q}$ is defined uniquely in terms of the mode-coupling functional $\mathcal{F}_{q}$. This means that $F_{q}(t)$ is fixed via equations (2) by the equilibrium structure. In particular, $F_{q}$ is independent of $\Omega_{q}$ and $M_{q}^{\text {reg }}(t)$. This result implies, for example, that the glassy dynamics is the same for all hard-sphere systems, provided times are measured in the units of $t_{0}$. It is the same both for the HSS obeying the Newtonian microscopic dynamics and for a system exhibiting the Brownian singleparticle motion, like in a colloid. And it is the same for a HSS model where the 
spheres move in elementary steps, as used in a Monte-Carlo simulation. Thus, the MCT provides us with understanding why the glassy dynamics observed in colloids [29] exhibits in all details the same scenario as the one observed in more conventional systems, like CKN $[51,54]$. One can also show that $F_{q}$ deals with relaxation in the sense that it can be written as superposition of exponentials: $F_{q}(t)=\int_{0}^{\infty} \exp (-\gamma t) \rho_{q}(\gamma) \mathrm{d} \gamma$, where $\rho_{q}(\gamma) \geqslant 0$. In this sense it is appropriate to refer to the dynamics outside the transient as structure relaxation. The preceding statements are discussed and demonstrated in more detail in [22].

The scale $t_{\sigma}$ characterizes the MCT- $\beta$-process window; it is the scale entering the first scaling law, equations (10). For times of order $t_{\sigma}$, the glass correlator comes close to its long-time asymptote $f_{q}$ and the liquid correlator crosses the plateau $f_{q}^{c}$ (compare the $n=9$ curves in figure 1). Equivalently, for the frequency of order $1 / t_{\sigma}$ the glass-state susceptibility spectrum $\chi_{q}^{\prime \prime}(\omega)$ exhibits a knee due to the crossover from a sublinear to a linear variation. The liquid susceptibility exhibits a minimum for the frequency of order $1 / t_{\sigma}$ due to the crossover from the von Schweidler law to the critical law (compare the $n=9$ curves in figure 3 ). These statements hold in the regime where $|\sigma|$ is so small that the leading-orderasymptotic results are valid; for larger $|\sigma|$ the results for the $\beta$-dynamics can be more complicated [23].

The scale $t_{\sigma}^{\prime}$ characterizes the MCT- $\alpha$-process window; it is the scale entering the second scaling law, equation (14). For times of order $t_{\sigma}^{\prime}$, the glass correlators differ from their long-time asymptote only by exponentially small terms, and the liquid correlators decrease to, say, $f_{q}^{\text {c }} / 2$ (compare the $n=9, \epsilon<0$ curve in figure 1). The frequency $1 / t_{\sigma}^{\prime}$ is the scale for the $\alpha$-peak position of the susceptibility spectrum (compare the $n=9, \epsilon<0$ curve in figure 3 ). This frequency marks a crossover of the strong quasielastic fluctuation spectrum to a frequency independent behaviour (compare the $n=9, \epsilon<0$ curve in figure 2).

Both scales, $t_{\sigma}$ and $t_{\sigma}^{\prime}$, diverge upon approaching the critical point; but according to equations (11), the ratio diverges for $|\sigma| \rightarrow 0$ as well:

$$
t_{\sigma} / t_{0} \rightarrow \infty, t_{\sigma}^{\prime} / t_{0} \rightarrow \infty, t_{\sigma}^{\prime} / t_{\sigma} \rightarrow \infty
$$

In this sense there are two critical scales. It is this finding which makes the slow MCT dynamics so subtle and different compared to the one observed for conventional fold bifurcations or for second order phase transitions. The latter theories deal with a single critical scale only, which is the analogue of $t_{\sigma}$. Let us also note, that the window for the MCT- $\beta$-process, $t_{0} \ll t \ll t_{\sigma}^{\prime}$, overlaps with the window for the MCT- $\alpha$-process, $t_{\sigma} \ll t$. Within the overlap window, $t_{\sigma} \ll t \ll t_{\sigma}^{\prime}$, the long-time asymptote of the first scaling law, equation (10a) for $t / t_{\sigma}=\hat{t} \gg 1$, is identical with the short-time asymptote of the second scaling law, equation (14) for $t / t_{\sigma}^{\prime}=\tilde{t} \ll 1$, and this is von Schweidler's law. The divergence of the ratio $t_{\sigma}^{\prime} / t_{\sigma}$ is a prerequisite for the proof of equation $(7 \mathrm{~b})$.

The increase of $t_{\sigma}, t_{\sigma}^{\prime}$ and $t_{\sigma}^{\prime} / t_{\sigma}$ upon cooling CKN or compressing the HSS can be inferred from a look at the raw data published for the spectra [51] or decay curves [29], respectively. In the both references [29,51] the data were shown 


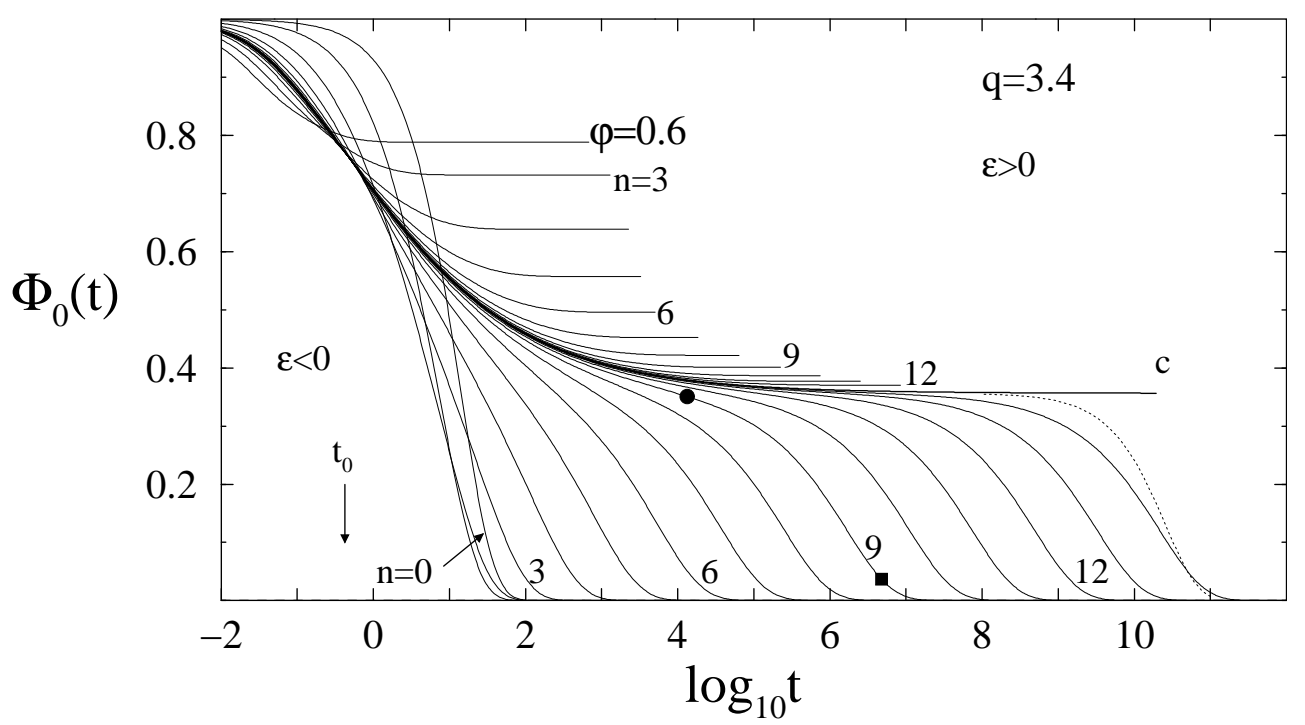

Figure 1. Density correlator $\phi_{0}(t)$ for the HSS for wave vector $q d=3.4$ and packing fractions $\varphi / \varphi_{\mathrm{c}}=1+\epsilon, \epsilon= \pm 10^{-n / 3}$; see text. The heavy line with label $\mathrm{c}$ is the solution for $\varphi=\varphi_{\mathrm{c}}$. The dotted line denotes $\phi_{\mathrm{D}}=f^{\mathrm{c}} \exp \left[-\left(t / \tau_{\mathrm{D}}\right)\right]$ with $f^{\mathrm{c}}=0.356, \tau_{\mathrm{D}}=2.56 \cdot 10^{10}$. The full dot and square mark the times $t_{\sigma}$ and $t_{\sigma}^{\prime}$, respectively, for $\epsilon=-0.001$ [64].

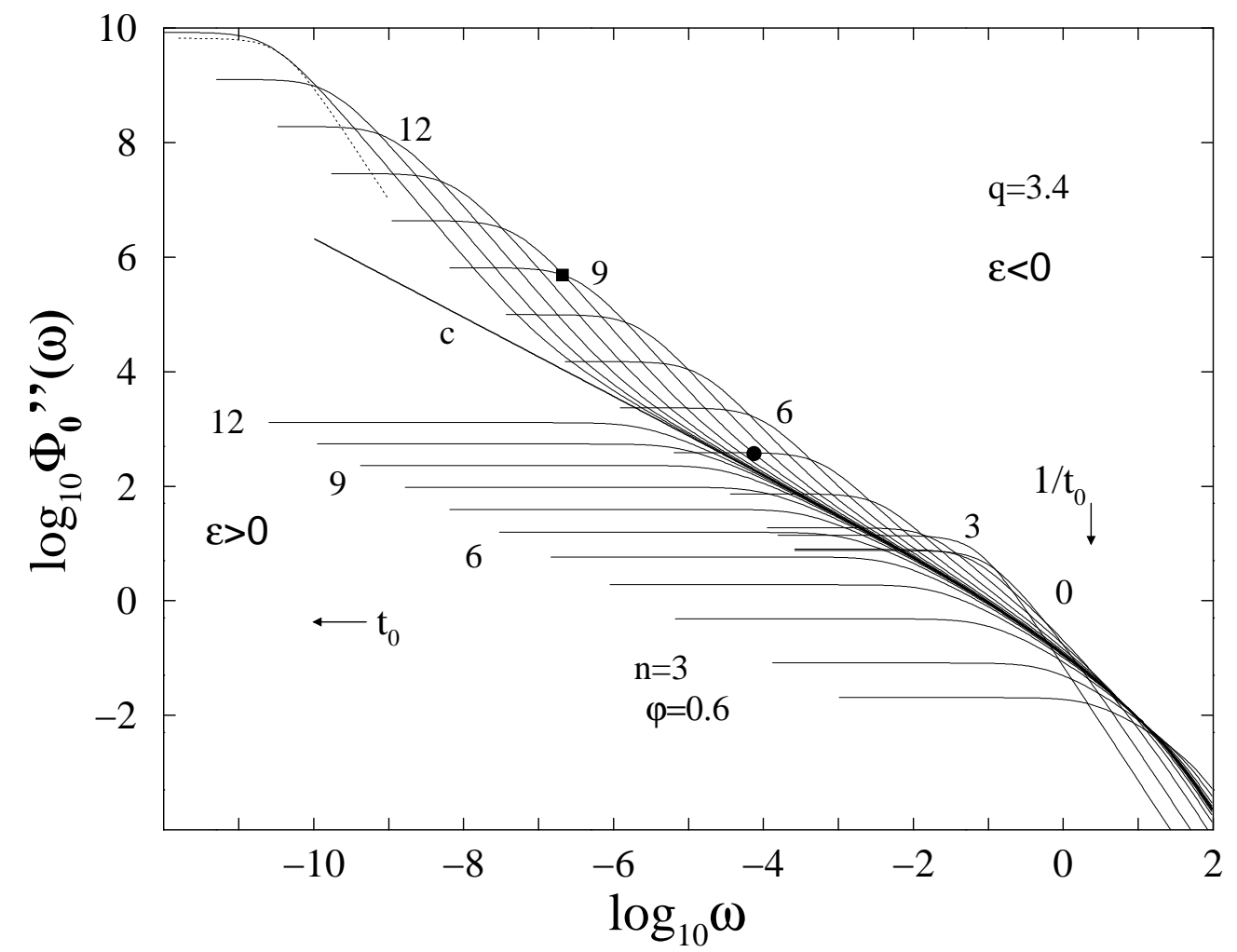

Figure 2. Correlation spectra $\phi_{0}^{\prime \prime}(\omega)$ for the results of figure 1 . The dotted curve is $\phi_{\mathrm{D}}^{\prime \prime}(\omega)=2 \chi_{\max } \tau_{\mathrm{D}} /\left[1+\left(\omega \tau_{\mathrm{D}}\right)^{2}\right]$ with $\chi_{\max }=0.130$. The full dot and square mark the frequencies $1 / t_{\sigma}$ and $1 / t_{\sigma}^{\prime}$, respectively, for $\epsilon=-0.001$ [64]. 


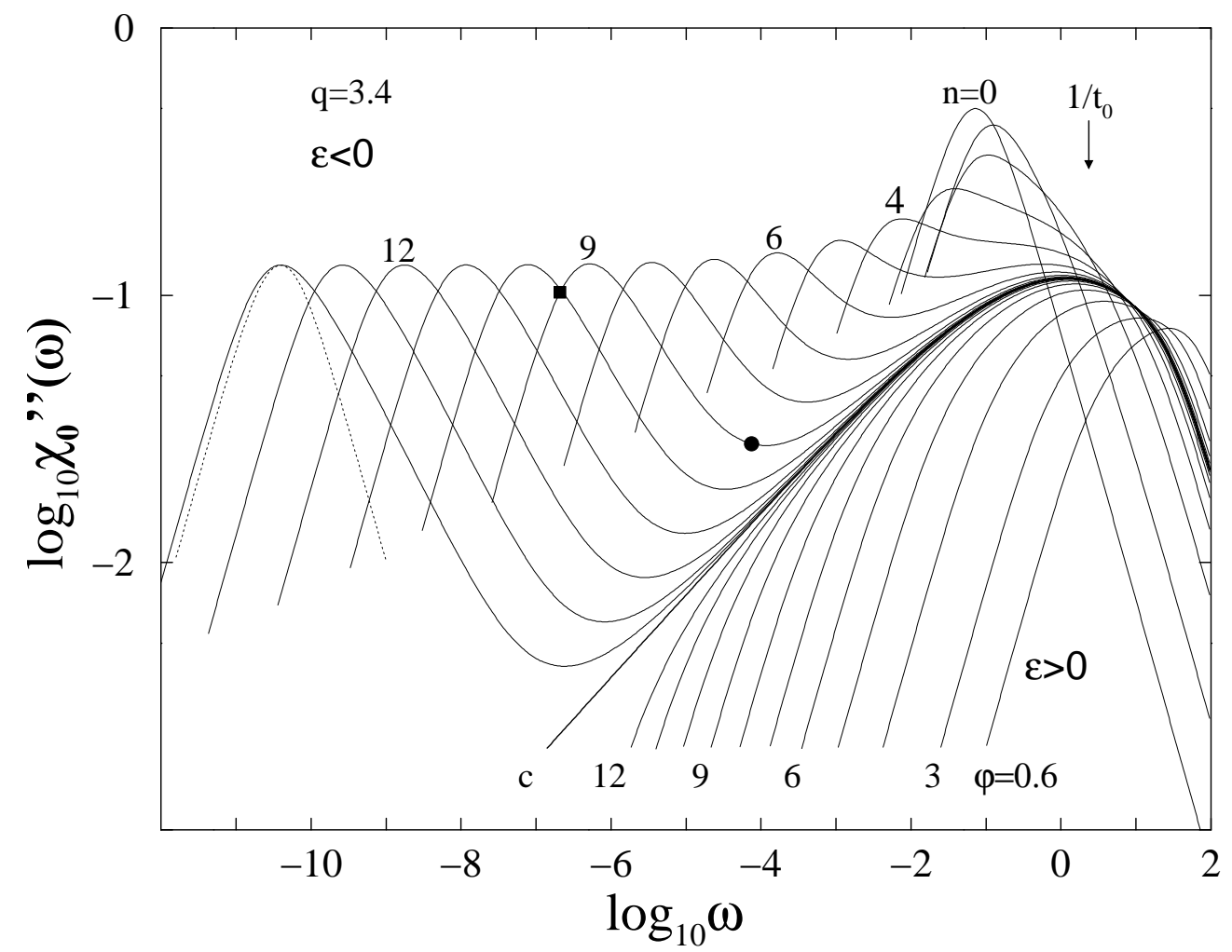

Figure 3. Susceptibility spectra $\chi_{0}^{\prime \prime}(\omega)=\omega \phi_{0}^{\prime \prime}(\omega)$ for the results shown in figure 2 . The dotted curve denotes $\chi_{D}^{\prime \prime}(\omega)=\omega \phi_{D}^{\prime \prime}(\omega)[64]$.

to be consistent with the predicted power laws, equations (11). An impressive demonstration of equations (16) was reported by Bartsch et al. [63] for a certain colloidal suspension. These authors measured the anomalous dynamics by a photon-correlation spectroscopy for a window as huge as seven orders of magnitude. The decay of the correlators $\phi_{q}(t)$ from 0.9 to 0.2 could be fitted with a high precision by the first scaling law (10a) for $\lambda=0.88$. The two measured scales $t_{\sigma}$, $t_{\sigma}^{\prime}$ followed the power laws, equations (11), with the exponents $1 / 2 a=2.2$ and $\gamma=3.6$ corresponding to the cited exponent parameter $\lambda$. For the effective packing fraction $\varphi=0.50$ the ratio $t_{\sigma}^{\prime} / t_{\sigma}=10$ was found. The ratio increased with an increase of $\varphi$ to $t_{\sigma}^{\prime} / t_{\sigma}=1000$.

Near the critical point and outside the transient regime the MCT dynamics of a liquid exhibits a two-step-relaxation scenario. The first step for $t_{0} \ll t<t_{\sigma}$ deals with the critical decay towards the plateau $f_{q}^{c}$. The second step is the $\alpha$-decay from the plateau to zero. The start of the $\alpha$-process is not identical with the end of the regular transient dynamics as it was anticipated in the earlier literature; rather it is time $t_{\sigma}$, which itself increases singularly upon cooling or compressing the system. 


\section{Evolution of structural relaxation: An idealized description}

Figures 1-3 [64] exhibit evolution of the bifurcation dynamics for a hard-spherecolloid model, equations $(2-4)$, for the wave vector $q d=3.4$. The wave vectors were discretized to $M=100$ equally spaced values, the structure factor $S_{q}$ was calculated within the Percus-Yevick theory, the time unit was chosen such that $\nu(d / v)^{2}=160$. More details and figures for other wave vectors can be found in [23]. For $\epsilon=-0.01$ the shown correlator $\phi_{0}(t)$ needs an increase of time $t$ by more than the factor $10^{5}$ for its decay from 0.9 to 0.1 . The upper half of the corresponding susceptibility spectrum, the $n=6(\epsilon<0)$ curve in figure 3, also extends over a window of more than 5 decades. To exhibit this enormous relaxation stretching in a diagram, a linear-time or linear-frequency axis cannot be used, it is a tradition to use a logarithmic abscissa.

The $\alpha$-relaxation parts of the curves in figure 1, i.e. the decay curves from the plateau value $f^{c}=0.356$ to zero, are related for $n \geqslant 6$ by shifts parallel to the $\log t$-abscissa. These relaxation curves cause the low frequency $\alpha$-peaks for the susceptibility spectra in figure 3 , which can also be superimposed by shifts parallel to the $\log \omega$-abscissa. These shift-laws are equivalent to the second scaling law, equation (14). Dotted lines have been added in the figures to match the $n=14 \alpha$ process by the Maxwell-Debye-relaxation curves. The actual decay curve in figure 1 is flatter than the dotted exponential and the $\alpha$-peak in figure 3 is broader than the dotted Lorentzian. These observations demonstrate the stretching of the MCT $\alpha$-process.

The susceptibility spectra in figure 3 are shown with a logarithmic vertical axis, so that power laws $\chi^{\prime \prime}(\omega) \propto \omega^{x}$ can be easily identified as straight lines with slope $\mathrm{x}$. For large $n$-curves, one recognizes for the high-frequency- $\alpha$-peak wing the von Schweidler-power-law asymptote with $x=-b=-0.58$, equation (7b). The high-frequency parts of the spectral minimum approach the critical law with $x=a=0.31$, equation (6). The susceptibility minima in a double logarithmic plot shift down with decreasing $|\epsilon|$, parallel to the critical spectrum, without change of the shape. This shift law is equivalent to the first scaling law, equation (10a).

In the figures the distance parameter is changed in a geometrical progression: $\epsilon=\left(\varphi-\varphi_{\mathrm{c}}\right) / \varphi_{\mathrm{c}}= \pm 10^{-n / 3}, n=0,1, \ldots$ Successive positions of the $\alpha$-peak frequency, as well as of the position and intensity of the susceptibility minima, differ by the same shift values. This is equivalent to the statement that the scales for $1 / t_{\sigma}^{\prime}, 1 / t_{\sigma}$ and $c_{\sigma}$ follow power laws $|\epsilon|^{\gamma},|\epsilon|^{1 / 2 a}$ and $|\epsilon|^{1 / 2}$, respectively, where the HSS values for the exponents are $1 / 2 a=1.60, \gamma=2.46$. One notices that with increasing $n$ the positions of the maxima shift more than the ones for the minima. This is equivalent to the increase of $t_{\sigma}^{\prime} / t_{\sigma}$, equation (16).

A detailed analysis of the shown numerical solutions of the MCT equations brings out the following [23]. The various leading-order-asymptotic results, which were discussed in the preceding sections 3-7, describe the correlators and spectra within the structural relaxation window qualitatively for $|\epsilon| \leqslant 0.01$ and yield a 
quantitative description for $|\epsilon| \leqslant 0.001$. If the leading-order corrections are incorporated, the quantitative description is extended up to $|\epsilon| \cong 0.01$; and also the qualitative deviations of the solutions from their leading-order-asymptotic description can be understood. This holds for times down to and for frequencies up to the regime, where microscopic transient effects start to play a major role. In this sense one concludes that the MCT solutions for the bifurcation dynamics are understood. At present it is not clear whether the cited statements for the HSS are also valid for other systems. None of the cited analytical results deals with the crossover phenomena from structural relaxation to transient dynamics. For conventional systems, obeying the Newtonian dynamics, the crossover phenomena can be quite different from those for colloids, obeying the Brownian dynamics. This is known from some numerical examples [22]. The cross-over dynamics, which is of an obvious relevance for a possible application of MCT to the glassy dynamics in liquids, has not yet been studied comprehensively.

Several strategies have been followed to test the applicability of the MCT for the description of the glassy-dynamics evolution. The most obvious approach is a check whether the experimental data follow qualitatively the universal patterns obtained from the leading asymptotic solutions for temperatures $T$ near the critical value $T_{\mathrm{c}}$. This can be done best by fitting the data to the various laws discussed above, using theoretically well defined quantities like $\lambda, h_{A}$ etc. as fit parameters. The major problem with that approach is, that there is no a priori knowledge about the size of the dynamical window and of the temperature interval where the leading formulae should work. It is crucial, therefore, at least to test that the fitting intervals expand with the decreases of $\left|T-T_{\mathrm{c}}\right|$, as requested by the theory. Such data discussions have been done, for example, for CKN [41,51-53], OTP $[36,59]$, two colloids $[29,63]$, the molecular dynamics data for a binary mixture [3133] and water [57], and the Monte-Carlo data for a polymer model [65]. Within a refined version of this strategy one fits the whole structural relaxation dynamics by splicing together the results of the first and the second scaling laws. This restricts the choice of fitting parameters. Cummins et al. [59] demonstrated such an analysis for their depolarized-light-scattering spectra of OTP for the three decade frequency window between $0.1 \mathrm{GHz}$ and $100 \mathrm{GHz}$ and for the temperatures between $320 \mathrm{~K}$ and $415 \mathrm{~K}$. Their work implies, in particular, the identification of the $\alpha$-process, the verification of the time-temperature-superposition principle and the proper description of the $\alpha-\beta$-relaxation interplay for the temperatures as high as $40 \mathrm{~K}$ above the melting temperature $T_{\mathrm{m}}$.

The second strategy uses the results for schematic models with smoothly drifting parameters. There are two advantages of this approach: one does not rely on asymptotic expansions, and one can also study the crossover from the relaxation to oscillation dynamics. The drawback of this approach seems to be, that there is much freedom in the choice of the model and in the decision which parameters are allowed to drift and which are kept fixed. The examples of such studies are the interpretation of light-scattering spectra of glycerol [66] and CKN [67] with a two-component model defined by equations (12). 
The most ambitious approach aims at understanding all structural relaxation features within a microscopic frame. A prerequisite for this strategy is a good understanding of the equilibrium structure so that the mode-coupling functional, equations (2), can be quantified. The possibility of such undertaking was demonstrated in the cited work on hard sphere colloids [29,30]. Van Megen and Underwood spliced together the calculated HSS- $\alpha$-relaxation master functions $\tilde{\phi}_{q}(\tilde{t})$ and the first scaling law result, equations (8-11), for the predicted exponent parameter $\lambda$. As fit quantities entered the microscopic time scale $t_{0}$, the separation parameter $\sigma$, the critical Debye-Waller factor $f_{q}^{c}$, and the critical amplitude $h_{q}$. After the fit, they observed that $t_{0}$ was a $\sigma$ - and $q$-independent constant and also that $f_{q}^{\mathrm{c}}, h_{q}$ agreed with the MCT prediction for a representative set of wave vectors. Then they showed that $\sigma$ followed the predicted law $\sigma=C\left(\varphi-\varphi_{\mathrm{c}}\right) / \varphi_{\mathrm{c}}$ with the predicted guage factor $C$. The fitted value for $\varphi_{\mathrm{c}}$ differed from the predicted one by about $12 \%$. Thus, the interpretation of their results could be done quantitatively using $t_{0}$, given by the viscosity of the solvent, as a free fit parameter and adjusting the critical-point position $\varphi_{\mathrm{c}}$ a bit.

\section{Evolution of structural relaxation: An extended description}

A derivation of approximate equations of motion for the glassy dynamics was proposed by Das and Mazenko within a diagrammatic classification of a systematic perturbation expansion for a non-linear-hydrodynamics model [68]. Closed MCT equations were obtained as results of a self-consistent-one-loop treatment for the self-energy kernels. These equations lead to an ideal glass transition. However, incorporating two-loop terms they obtained an improved theory for which they could show that there is no ideal glass transition anymore. Unfortunately, compelling conclusions for the physics of liquids cannot be drawn from [68]. The vertices in the mode-coupling kernels are treated crudely, so that unspecified cut-off wave vectors have to be introduced. This excludes the possibility to evaluate critical temperatures, form factors or anomalous exponents. Moreover, it was claimed continuously $[68,69]$ as the main result of the fluctuating-hydrodynamics theory that the spikes of the ideal-glass-state spectra $\phi_{q}^{\prime \prime}(\omega)=\pi f_{q} \delta(\omega)+\cdots$ are transformed for $T \approx T_{\mathrm{c}}$ to the peaks of a diffusive type: $\phi_{q}^{\prime \prime}(\omega)=\gamma q^{2} /\left[\omega^{2}+\left(\gamma q^{2}\right)^{2}\right]+\cdots$. Apparently, the authors have not been aware that such a result contradicts the experimental facts: from the Brillouin-scattering and photon-correlation spectroscopy it is well known that $\alpha$-relaxation peaks for the density-fluctuation spectra are $q$-independent for small wave vectors $q$.

An extended MCT was derived [70] within a generalized-kinetic-equation approach [15] towards the dynamics of simple liquids. The basic version of the MCT, defined by equations (1-3) and referred to in this context as an ideal or simplified MCT, is obtained if only leading-order contributions to the anticipated slowing down of structure fluctuations are incorporated. These terms are due to the coupling of force fluctuations to density-fluctuation pairs, as described by equation 
(2a). The leading improvement of this approximation is equivalent to including the coupling of forces to current fluctuations, too. The current correlators are the analogues of phonon propagators studied in the theory of crystals. These new mode-coupling kernels provide contributions to phonon-assisted transport, so that these modifications of the theory are referred to as hopping effects. The extended MCT confirms the finding of [68] concerning the elimination of the ideal transition, but it does not suffer from the shortcomings specified in the preceding paragraph. The derivation of the extended MCT was reconsidered recently [71] within a perturbation-expansion approach for correlators.

The extended MCT equations can again be solved in the leading order of an expansion in $\delta \phi_{q}(t)=\phi_{q}(t)-f_{q}^{\mathrm{c}}$. This solution deals with an intermediate dynamical window extending from the end of the transient up to and including the start of the $\alpha$-process. Such a window exists in the limit of small separations $\sigma \propto T_{\mathrm{c}}-T$ provided the mentioned current couplings are small. As a result, one reproduces the factorization theorem, equation (8), where equation (9) for the $\beta$-correlator $G$ is to be extended to [70]:

$$
-\delta t+\sigma+\lambda G(t)^{2}=(\mathrm{d} / \mathrm{d} t) \int_{0}^{t} G\left(t-t^{\prime}\right) G\left(t^{\prime}\right) \mathrm{d} t^{\prime} .
$$

Here $\lambda$ is the exponent parameter introduced above, and also $\sigma$ has the same values as in the ideal MCT. The number $\delta \geqslant 0$, which is called a hopping parameter, has to be evaluated from the new relaxation kernels. It vanishes only if all the current couplings are zero, i.e. if the idealized MCT is considered. The $\beta$-correlator is now a function of the two parameters $\sigma$ and $\delta \cdot t_{0}: G(t)=g\left(t / t_{0}, \sigma, \delta \cdot t_{0}\right)$. The function $g$ is homogeneous: $g\left(x \cdot \xi, y \cdot \xi^{2 a}, z \cdot \xi^{1+2 a}\right)=\xi^{a} g(x, y, z)$ for all $\xi>0$, a property also referred to a two-parameter scaling. Thus, the $\beta$-dynamics has to be discussed in the half-plane of the parameter points $\left(\sigma, \delta \cdot t_{0}\right), \delta \geqslant 0$. The glass transition singularity is located at the origin $\left(\sigma=0, \delta \cdot t_{0}=0\right)$ and here the correlator is given by $\left(t_{0} / t\right)^{a}$. The ideal glass states are located on the half-abscissa $\left(\sigma \geqslant 0, \delta \cdot t_{0}=0\right)$.

Upon lowering the temperature, $\sigma$ increases and $\delta$ decreases. The system moves along a path from the states with large negative $\sigma$ and large $\delta$ to the states with large positive $\sigma$ and small $\delta$. The latter states are close to ideal glass states. The glass-transition singularity is avoided, the solutions always describe a relaxation towards the equilibrium. However, temperature $T_{\mathrm{c}}$ marks a crossover from glassyliquid states for $T \gg T_{\mathrm{c}}$ to almost arrested states for $T \ll T_{\mathrm{c}}$. The former can be described by ignoring $\delta$, i.e. by the ideal MCT. In this limit the transport coefficients are mainly due to interaction effects between the density fluctuations. For $T \ll T_{\text {c }}$ the transport coefficients and the $\alpha$-processes in general are due to the hopping effects, and therefore cannot be described by the ideal MCT. But also for $T<T_{\mathrm{c}}$ there is a dynamical window, where the dynamics can still be described with the $\delta=0$ solutions. The solutions of equation (17) are well understood [72]. But so far it has not been possible to solve the complete extended MCT equations for some fluid model. Solutions of equation (17) have been used for a quantitative 
analysis of depolarized-light-scattering spectra of CKN and Salol [52], of propylene carbonate [73] and of orthoterphenyl [59]. Also Monte-Carlo simulation data for a polymer model could be interpreted quantitatively within the extended MCT scenario [74].

\section{Concluding remarks}

Already in 1969 M.Goldstein argued [75] that there is a crossover temperature $T_{\mathrm{c}}$ separating the equilibrium states of cooled or compressed liquids in two regimes of quite different dynamical behaviours. He conjectured, in particular, that interesting phenomena are connected with the crossover, which show up for the spectra in the GHz-window. However, only after the invention of the MCT the experiments were reported which substantiated Goldstein's statements. This theory gave a precise meaning to the concept of $T_{\mathrm{c}}$, and predicted in a rather specific manner, which characteristic crossover phenomena should be observable.

An essential outcome of the MCT is a prediction of bifurcation points, called glass transition singularities. This implies a possibility to introduce a pair of small parameters $(\sigma, \delta)$ specifying the distance of states from the singularity $(\sigma=0, \delta=$ $0)$. Asymptotic expansions can then be carried out to solve analytically the complicated equations of motion within a very interesting region near the singularity. Thereby, an understanding of the general features of the glassy dynamics can be achieved. It turned out that the MCT bifurcation dynamics is quite different from what one knows from other theories of condensed matter or from other singularity theories. This is an obvious necessity for any successful theory of structural relaxation, since one knows for more than a century that the experimental findings for glassy systems are quite different from the ones for other condensed matter. Some of the non-trivial results of the MCT were described in the preceding sections; and for each of them some modern experiment was quoted, which provided evidence for the experimental relevance of the results. Furthermore, it can be noted that up to now no alternative explanations for the above cited experimental findings have been reported. It might also be adequate to emphasize that the MCT suggested solutions to some ancient puzzles of the glassy dynamics, such as the existence of stretched $\alpha$-peaks, von Schweidler's power-law decay and Kohlrausch's law.

The dynamics of simple liquids in their normal state, say argon near its triple point, was studied extensively in the past by neutron-scattering experiments and by molecular-dynamics simulations. It is understood that this dynamics can be described by proper modifications of Enskog's kinetic equation. Alternatively, one can write down the exact representations of the correlation functions in terms of relaxation kernels and model the latter, so that the basic sum rules are taken into account. This work corroborated Goldstein's reasoning [75] concerning the irrelevance of the transition-state theory to the explanation of transport coefficients for the normal-liquid state. One cannot understand viscosities, diffusivities and the like for $T>T_{\mathrm{c}}$ within a physical framework, which was developed to justify the Arrhenius law. In particular, these studies brought out the crucial role of the cage effect 
[76]. About twelve nearest neighbours of a tagged particle form a cage. If these cage-forming particles were fixed, the tagged particle could not leave this cage, only a rattling motion within the cage would be allowed. The motion of a tagged particle over distances of the particle diameter requires rearrangements of many other particles. A long distance motion is therefore a cooperative phenomenon. The particles forming the cage move in a similar manner as the particle trapped in the cage. One should not approach the problem with ad-hoc assumptions for the cage motion. Rather one has to solve the problem of the cage formation simultaneously with the problem of the motion within the cage. The idealized MCT focuses on this self-consistency problem. The first equation (1) describes in a well-known fashion the motion within a given cage. The motion is ruled by the kernel $M_{q}(t)$; it deals with the result of the forces on the tagged particle produced by the cageforming neighbours. The second equation (2) describes how the forces fluctuate due to the motion of the particles which form the cage.

As a result, one obtains a well-defined theory for Goldstein's view. For $T>T_{\mathrm{c}}$ liquid transport like diffusion is possible. The tagged particle moves because its neighbour moves out of the way. This neighbour can move because its neighbours move, etc. However, for $T<T_{\mathrm{c}}$ liquid transport via such streaming with backflow patterns is impossible. The tagged particle cannot move because its cage-forming neighbours cannot move. These neighbours cannot move because their neighbours cannot move, etc. Within the ideal MCT the particles arrest for $T<T_{\mathrm{c}}$ in a random array spanning a frozen potential landscape. The latter is of such a structure that percolation is impossible. One gets an ideal glass state. Incorporating hopping effects within the extended MCT, diffusion becomes possible via the same processes as often discussed for crystalline matter. Since the ideal glass state exhibits no longranged order, the possibility for hopping implies the possibility for a slow decay of all cages. Due to hopping one comes back to liquid transport also for $T<T_{\mathrm{c}}$, albeit via an utterly different mechanism than for $T>T_{\mathrm{c}}$. The extended MCT allows one to follow the evolution of structural relaxation patterns also for temperatures near the crossover value $T_{\mathrm{c}}$. But at present it does not seem justified to assume that the extended MCT describes the $\alpha$-process also for temperatures far below $T_{\mathrm{c}}$, say for $T$ near $T_{\mathrm{g}}$. In this low-temperature regime $\alpha$-relaxation occurs on time scales which are 6 or more orders of magnitude below the microscopic scale for the dynamics.

For temperatures far below $T_{\mathrm{c}}$ the dynamics deals with the generalized Brownian motion, i.e. with the percolation problem of thermally activated particles in a random-potential landscape. This problem is studied extensively in various theories of disordered semiconductors. A rather general outcome of these theories is the prediction that transport coefficients vary according to the $\tau \propto \exp \left(T_{0} / T\right)^{2}$ law. This law also describes $\alpha$-relaxation times $\tau$ in glassy liquids over many orders of magnitude, say for $\tau$ between $10^{2}$ and $10^{-4}$ sec. This formula can be used to explain viscosity variations for temperatures increasing from $T_{\mathrm{g}}$ to some upper limit $T_{\mathrm{c}}^{*}$ near $T_{\mathrm{c}}$, as can be inferred from [77] and the papers quoted there. The MCT has provided justification of the underlying picture of an almost frozen random poten- 
tial, it quantified the almost frozen array of particles by its Debye-Waller factor, and explained in detail the dynamics of the melting of the array for temperatures increasing towards $T_{\mathrm{c}}$.

The classical experiments [1] dealt with the glassy dynamics, where the $\alpha$ relaxation time is between six and twelve orders of magnitude larger than the microscopic time scale for motion in condensed matter. Therefore, the temperature had to be lowered considerably below $T_{\mathrm{c}}$, and in most cases the system is then in a metastable supercooled state. Usually the true equilibrium state is a crystalline solid. But the MCT predicted that the $\alpha$-process with its puzzling features is fully developed considerably above $T_{\mathrm{c}}$, where it can be measured in the $\mathrm{GHz}$ window. This implies that for the evolution of the glassy dynamics it is irrelevant whether or not the system is supercooled or supercompressed. What matters is that there is an amorphous structure which is sufficiently dense. Then, interaction effects on length scales of the particle diameters render the cage effect so important that the ideal-glass-transition precursors dominate the whole slow particle motion. The demonstration that in conventional glass-forming liquids like OTP [59], salol $[78,79]$ and glycerol $[80,66,40]$ the $\alpha$-process is fully developed for temperatures considerably above the melting point $T_{\mathrm{m}}$, supports this MCT implication.

A certain degree of complexity of the structure is necessary to keep a system in a metastable amorphous state. Simple systems crystallize too easily for $T<T_{\mathrm{m}}$. For example, for a binary Lennard-Jones mixture the pair potentials have to be chosen carefully to avoid nucleation effects for $T$ near $T_{\mathrm{c}}$ [32]. A system of hard spheres, where all particles have the same diameter, crystallizes no matter how fast the sample is produced for a packing fraction $\varphi$ above the freezing point $\varphi_{\mathrm{f}}=$ 0.49 [81]. However, introducing a random distribution for the particle diameters increases the nucleation time dramatically. Polydispersity of the order of $10 \%$ is sufficient to prevent nucleation for practically arbitrary long times [82]. It is an essential result of the MCT that complexity of the structure is irrelevant to the appearance of the glassy dynamics. The MCT results, as opposed to the results measured for nucleation rates, are robust to fine-tuning effects for the structure. A polydispersity of $10 \%$ will cause changes of a similar size for the structure factor $S_{q}$, and this will lead via equations (1-3) to small changes of the critical packing fraction $\varphi_{\mathrm{c}}$, amplitudes $f_{q}^{\mathrm{c}}, h_{q}$ and the like, without changing the general pattern calculated for a monodisperse system. The discovery of the ideal-glass transition for a polydisperse hard-sphere system [43] supports this MCT result.

\section{References}

1. Wong J., Angell C.A. Glass: Structure by Spectroscopy. Marcel Dekker, Inc., New York, 1976.

2. Menon N., O’Brien K.P., Dixon P.K., Wu L., Nagel S.R., Williams B.D., Carini J.P. Wide-frequency dielectric susceptibility measurements in glycerol. // J. Non-Cryst. Solids, 1992, vol. 141, p. 61-65. 
3. Mezei F., Knaak W., Farago B. Neutron spin-echo study of dynamic correlations near the liquid-glass transition. // Phys. Rev. Lett., 1987, vol. 58, p. 571-574.

4. Mezei F., Knaak W., Farago B. Neutron spin-echo study of dynamic correlations near liquid-glass transition. // Phys. Scr. T, 1987, vol. 19, p. 363-368.

5. Proceedings of the International Discussion Meeting on Relaxation in Complex Systems. Heraklion, Greece. Edited by Ngai K.L., Wright G.B., J. Non-Cryst. Solids 1991, vol. 131-133, p. 1-1285.

6. Proceedings of the Second International Discussion Meeting on Relaxation in Complex Systems. Alicante, Spain. Edited by Ngai K.L., Riande E., Wright G.B., J. Non-Cryst. Solids, 1994, vol. 172-174, p. 1-1457.

7. Proceedings of the Third International Discussion Meeting on Relaxation in Complex Systems. Vigo, Spain. Edited by Ngai K.L., Wright G.B., J. Non-Cryst. Solids, 1998, vol. 235-237, p. 1-814.

8. Zubarev D., Morozov V., Röpke G. Statistical Mechanics of Nonequilibrium Processes, vol. 2. Akademie Verlag, Berlin, 1996.

9. Kawasaki K. Kinetic equations and time correlation functions of critical fluctuations. // Ann. Phys. (N.Y.), 1970, vol. 61, p. 1-56.

10. Barrat J.-L., Latz A. Mode coupling theory for the glass transition in a simple binary mixture. // J. Phys.: Condens. Matter, 1990, vol. 2, p. 4289-4295.

11. Fuchs M., Latz A. $\alpha$-relaxation in a supercooled binary mixture. // Physica A, 1993, vol. 201, p. 1-13.

12. Schilling R., Scheidsteger T. Mode coupling approach to the ideal glass transition of molecular liquids: Linear molecules. // Phys. Rev. E, 1997, vol. 56, p. 2932-2949.

13. Franosch T., Fuchs M., Götze W., Mayr M.R., Singh A.P. Theory for the reorientational dynamics in glass-forming liquids. // Phys. Rev. E, 1997, vol. 56, p. 5659-5674.

14. Götze W., Lücke M. Dynamical structure factor $s(q, \omega)$ of liquid helium II at zero temperature. // Phys. Rev. B, 1976, vol. 13, p. 3825-3842.

15. Sjögren L. Kinetic theory of current fluctuations in simple classical liquids. // Phys. Rev. A, 1980, vol. 22, p. 2866-2882.

16. Leutheusser E. Dynamical model of the liquid-glass transition. // Phys. Rev. A, 1984, vol. 29, p. 2765-2773.

17. Bengtzelius U., Götze W., Sjölander A. Dynamics of supercooled liquids and the glass transition. // J. Phys. C, 1984, vol. 17, p. 5915-5934.

18. Götze W. Aspects of structural glass transitions. - In: Liquids, Freezing and Glass Transition. (Amsterdam, 1991), J.-P. Hansen, D.Levesque, and J.Zinn-Justin, Eds., North-Holland, p. 287-502.

19. Haussmann R. Some properties of mode coupling equations. // Z. Phys. B, 1990, vol. 79 , p. 143-148.

20. Götze W., Sjögren L. General properties of certain non-linear integro-differential equations. // J. Math. Analysis and Appl., 1995, vol. 195, p. 230-250.

21. Götze W., Sjögren L. Comments on the mode coupling theory for structural relaxation. // Chem. Phys., 1996, vol. 212, p. 47-59.

22. Franosch T., Götze W., Mayr M.R., Singh A.P. Structure and structure relaxation. // J. Non-Cryst. Solids, 1998, vol. 235-237, p. 71-85.

23. Franosch T., Fuchs M., Götze W., Mayr M.R., Singh A.P. Asymptotic laws and preasymptotic correction formulas for the relaxation near glass-transition singularities. // Phys. Rev. E, 1997, vol. 55, p. 7153-7176. 
24. Edwards S.F., Anderson P.W. Theory of spin glasses. // J. Phys. F, 1975, vol. 5, p. 965-974.

25. Arnol'd V.I. // Catastrophe Theory, 3rd ed. Springer-Verlag, Berlin, 1992.

26. Götze W. An elementary approach towards the Anderson transition. // Solid State Commun., 1978, vol. 27, p. 1393-1395.

27. Götze W., Leutheusser E., Yip S. Dynamical theory of diffusion and localization in a random, static field. // Phys. Rev. A, 1981, vol. 23, p. 2634-2643.

28. Götze W., Leutheusser E., Yip S. Correlation functions of the hard-sphere Lorentz model. // Phys. Rev. A, 1981, vol. 24, p. 1008-1015.

29. van Megen W., Underwood S.M. Glass transition in colloidal hard sphere: Measurement and mode-coupling-theory analysis of the coherent intermediate scattering function. // Phys. Rev. E, 1994, vol. 49, p. 4206-4220.

30. van Megen W. Crystallization and the glass transition in suspensions of hard colloidal spheres. // Transp. Theory Stat. Phys., 1995, vol. 24, p. 1017-1051.

31. Kob W., Anderson H.C. Scaling behavior in the $\beta$-relaxation regime of a supercooled Lennard-Jones mixture. // Phys. Rev. Lett., 1994, vol. 73, p. 1376-1379.

32. Kob W., Anderson H.C. Testing mode-coupling theory for a supercooled binary Lennard-Jones mixture: The van Hove correlation function. // Phys. Rev. E, 1995, vol. 51, p. 4626-4641.

33. Kob W., Anderson H.C. Testing mode-coupling theory for a supercooled binary Lennard-Jones mixture. II. Intermediate scattering function and dynamic susceptibility. // Phys. Rev. E, 1995, vol. 52, p. 4134-4153.

34. Nauroth M., Kob W. Quantitative test of mode-coupling theory for the ideal glass transition for a binary Lennard-Jones system. // Phys. Rev. E, 1997, vol. 55, p. 657667.

35. Petry W., Bartsch E., Fujara F., Kiebel M., Sillescu H., Farago B. Dynamic anomaly in the glass transition region of orthoterphenyl. A neutron scattering study. // Z. Phys. B, 1991, vol. 83, p. 175-184.

36. Tölle A., Schober H., Wuttke J., Fujara F. Coherent dynamic structure factor of orthoterphenyl around the mode coupling cross over temperature $T_{\mathrm{c}}$. // Phys. Rev. E, 1997, vol. 56, p. 809-815.

37. Yang Y., Nelson K.A. Impulsive stimulated thermal scattering study of $\alpha$ relaxation dynamics and the Debye-Waller factor anomaly in $\mathrm{Ca}_{0.4} \mathrm{~K}_{0.6}\left(\mathrm{NO}_{3}\right)_{1.4}$. // J. Chem. Phys., 1996, vol. 104, p. 5429-5436.

38. Knaak W., Mezei F., Farago B. Observation of scaling behaviour of dynamic correlations near liquid-glass transition. // Europhys. Lett., 1988, vol. 7, p. 529-536.

39. Tao N.J., Li G., Cummins H.Z. Self-similar light-scattering spectra of $\beta$ relaxation near the liquid-glass transition. // Phys. Rev. Lett., 1991, vol. 66, p. 1334-1337.

40. Schneider U., Lunkenheimer P., Brand R., Loidl A. Dielectric and far-infrared spectroscopy on glycerol. // J. Non-Cryst. Solids, 1998, vol. 235-237, p. 173-179.

41. Lunkenheimer P., Pimenov A., Loidl A. Fast dynamics in CKN and CRN investigated by dielectric spectroscopy. // Phys. Rev. Lett., 1997, vol. 78, p. 2995-2998.

42. Barrat J.-L., Götze W., Latz A. The liquid-glass transition of the hard-sphere system. // J. Phys.: Condens. Matter, 1989, vol. 1, p. 7163-7170.

43. van Megen W., Pusey P.N. Dynamic light-scattering study of the glass transition in colloidal suspensions. // Phys. Rev. A, 1991, vol. 43, p. 5429-5441.

44. Götze W., Sjögren L. $\beta$ relaxation of the glass transition of hard-spherical colloids. // 
Phys. Rev. A, 1991, vol. 43, p. 5442-5448.

45. Sciortino F., Gallo P., Tartaglia P., Chen S.-H. Supercooled water and the kinetic glass transition. // Phys. Rev. E, 1996, vol. 54, p. 6331-6343.

46. Gnedenko B.V., Kolmogorov A.N. // Limit Distributions for Sums of Independent Random Variables. Addison Wesley, Reading Mass., 1954.

47. Götze W. The scaling functions for the $\beta$-relaxation process of supercooled liquids and glasses. // J. Phys.: Condens. Matter, 1990, vol. 2, p. 8485-8498.

48. Götze W. Bifurcations of an iterated mapping with retardations. // J. Stat. Phys., 1996, vol. 83, p. 1183-1197.

49. Roux J.N., Barrat J.L., Hansen J.-P. Dynamical diagnostics for the glass transition in soft-sphere alloys. // J. Phys.: Condens. Matter, 1989, vol. 1, p. 7171-7186.

50. Signorini G.F., Barrat J.-L., Klein M.L. Structural relaxation and dynamical correlations in a molten state near the liquid-glass transition: A molecular dynamics study. // J. Chem. Phys., 1990, vol. 92, p. 1294-1303.

51. Li G., Du W.M., Chen X.K., Cummins H.Z., Tao N.J. Testing mode-coupling predictions for $\alpha$ and $\beta$ relaxation in $\mathrm{Ca}_{0.4} \mathrm{~K}_{0.6}\left(\mathrm{NO}_{3}\right)_{1.4}$ near the liquid-glass transition by light scattering. // Phys. Rev. A, 1992, vol. 45, p. 3867-3879.

52. Cummins H.Z., Du W.M., Fuchs M., Götze W., Hildebrand S., Latz A., Li G., Tao N.J. Light-scattering spectroscopy of the liquid-glass transition in $\mathrm{CaKNO}_{3}$ and in the molecular glass salol: Extended-mode-coupling-theory analysis. // Phys. Rev. E, 1993, vol. 47 , p. 4223-4239.

53. Fuchs M., Cummins H.Z., Du W.M., Götze W., Latz A., Li G., Tao N.J. Tests of the mode-coupling theory for a molten salt. // Phil. Mag. B, 1995, vol. 71, p. 771-781.

54. Mezei F. Scaling behaviour of structural relaxation near the glass transition: A critical analysis. // Ber. Bunsenges. Phys. Chem., 1991, vol. 95, p. 1118-1125.

55. Götze W. Some aspects of phase transitions described by the self consistent current relaxation theory. // Z. Phys. B, 1984, vol. 56, p. 139-154.

56. Sjögren L. Diffusion of impurities in a dense fluid near the glass transition. // Phys. Rev. A, 1986, vol. 33, p. 1254-1260.

57. Sciortino F., Fabbian L., Chen S.-H., Tartaglia P. Supercooled water and the kinetic glass transition. II. Collective dynamics. // Phys. Rev. E, 1997, vol. 56, p. 5397-5404.

58. Fuchs M., Hofacker I., Latz A. Primary relaxation in a hard-sphere system. // Phys. Rev. A, 1992, vol. 45, p. 898-912.

59. Cummins H.Z., Li G., Du W., Hwang Y.H., Shen G.Q. Light scattering spectroscopy of orthoterphenyl. // Prog. Theor. Phys. Suppl., 1997, vol. 126, p. 21-34.

60. Fuchs M., Götze W., Hofacker I., Latz A. Comments of the $\alpha$-peak shapes for relaxation in supercooled liquids. // J. Phys.: Condens. Matter, 1991, vol. 3, p. 5047-5071.

61. Götze W., Sjögren L. Relaxation processes in supercooled liquids. // Rep. Prog. Phys., 1992, vol. 55, p. 241-376.

62. Fuchs M. The Kohlrausch law as a limit solution to mode coupling equations. // J. Non-Cryst. Solids, 1994, vol. 172-174, p. 241-247.

63. Bartsch E., Antonietti M., Schupp W., Sillescu H. Dynamic light scattering study of concentrated microgel solutions as mesoscopic model of the glass transition in quasiatomic fluids. // J. Chem. Phys., 1992, vol. 97, p. 3950-3963.

64. Mayr M.R. PhD thesis, Physik-Department, Technische Universität München, 1998 (unpublished).

65. Baschnagel J. Analysis of the incoherent intermediate scattering function in the frame- 
work of the idealized mode-coupling theory: A Monte Carlo study for polymer melts. // Phys. Rev. B, 1994, vol. 49, p. 135-146.

66. Franosch T., Götze W., Mayr M.R., Singh A.P. Evolution of structural relaxation spectra of glycerol within the Gigahertz band. // Phys. Rev. E, 1997, vol. 55, p. 31833190.

67. Krakoviak V., Alba-Simionesco C., Krauzman M. Study of the depolarized light scattering spectra of supercooled liquids by a simple mode-coupling model. // J. Chem. Phys., 1997, vol. 107, p. 3417-3427.

68. Das S.P., Mazenko G.F. Fluctuating nonlinear hydrodynamics and the liquid-glass transition. // Phys. Rev. A, 1986, vol. 34, p. 2265-2282.

69. Kim B., Mazenko G.F. Fluctuating nonlinear hydrodynamics, dense fluids, and the glass transition. // Adv. Chem. Phys., 1990, vol. 78, p. 129-170.

70. Götze W., Sjögren L. The glass transition singularity. // Z. Phys. B, 1987, vol. 65, p. $415-427$.

71. Liu C. Z.-W., Oppenheim I. Reexamination of the mode-coupling scheme for the glass transition. // Physica A, 1997, vol. 235, p. 369-387.

72. Fuchs M., Götze W., Hildebrand S., Latz A. A theory for the $\beta$-relaxation process near the liquid-to-glass crossover. // J. Phys.: Condens. Matter, 1992, vol. 4, p. 7709-7744.

73. Du W.M., Li G., Cummins H.Z., Fuchs M., Toulouse J., Knauss L.A. Light-scattering study of the liquid-glass transition in propylene carbonate. // Phys. Rev. E, 1994, vol. 49, p. 2192-2205.

74. Baschnagel J., Fuchs M. Monte Carlo simulations of the glass transition in polymer melts: extended mode-coupling analysis. // J. Phys.: Condens. Matter, 1995, vol. 7, p. $6761-6780$.

75. Goldstein M. Viscous liquids and the glass transition: A potential energy barrier picture. // J. Chem. Phys., 1969, vol. 51, p. 3728-3739.

76. Balucani U., Zoppi M. Dynamics of the Liquid State. Clarendon Press, Oxford, 1994.

77. Richert R., Bässler H. Dynamics of supercooled melts treated in terms of the random walk concept. // J. Phys.: Condens. Matter, 1990, vol. 2, p. 2273-2288.

78. Li G., Du W.M., Sakai A., Cummins H.Z. Light-scattering investigation of $\alpha$ and $\beta$ relaxation near the liquid-glass transition of the molecular glass salol. // Phys. Rev. A, 1992, vol. 46, p. 3343-3356.

79. Torre R., Bartolini P., Pick R.M. Time-resolved optical Kerr effect in a fragile glassforming liquid, salol. // Phys. Rev. E, 1998, vol. 57, p. 1912-1920.

80. Wuttke J., Hernandez J., Li G., Coddens G., Cummins H.Z., Fujara F., Petry W., Sillescu H. Neutron and light scattering study of supercooled glycerol. // Phys. Rev. Lett., 1994, vol. 72, p. 3052-3055.

81. Rintoul M.D., Torquato S. Computer simulations of dense hard-sphere systems. // J. Chem. Phys., 1996, vol. 105, p. 9258-9265.

82. van Megen W., Underwood S.M., Müller J., Mortensen T.C., Henderson S.I., Harland J.L., Francis P. Particle diffusion and crystallization in suspensions of hard spheres. // Prog. Rep. Phys. Suppl., 1997, vol. 126, p. 171-180. 
Основні питання теорії взаємодіючих мод у динаміці скла

\section{В.Ґьотце}

Технічний університет м. Мюнхена, фізичний факультет

D-85747 Ґархінґ, Німеччина

Отримано 16 квітня 1998 р.

Пояснено суттєві результати теорії взаємодіючих мод для еволюції структурної релаксації в простих рідинах, зокрема, аномалію фактора Дебая-Уолера, критичний розпад, закон Швайдлера, скейлінг $\boldsymbol{\alpha}$-, $\boldsymbol{\beta}$-релаксації, появу двох розбіжних масштабів часу, закон Колрауша для $\alpha$-процесу. Також розповідається про їхнє значення для розуміння експериментів у склоутворюючих системах.

Ключові слова: теорія взаємодіючих мод, структурна релаксація, динаміка скла, динаміка біфуркації

PACS: $64.70 . P f, 61.20 . L C$ 